\title{
Interferometric Processing of Sentinel-1 TOPS Data
}

\author{
Nestor Yague-Martinez, Pau Prats-Iraola, Senior Member, IEEE, Fernando Rodriguez Gonzalez, \\ Ramon Brcic, Member, IEEE, Robert Shau, Senior Member, IEEE, Dirk Geudtner, \\ Michael Eineder, Senior Member, IEEE, and Richard Bamler, Fellow, IEEE
}

\begin{abstract}
Sentinel-1 (S-1) has an unparalleled mapping capacity. In interferometric wide swath (IW) mode, three subswaths imaged in the novel Terrain Observation by Progressive Scans (TOPS) SAR mode result in a total swath width of $250 \mathrm{~km} . \mathrm{S}-1$ has become the European workhorse for large area mapping and interferometric monitoring at medium resolution. The interferometric processing of TOPS data however requires special consideration of the signal properties, resulting from the ScanSAR-type burst imaging and the antenna beam steering in azimuth. The high Doppler rate in azimuth sets very stringent coregistration requirements, making the use of enhanced spectral diversity (ESD) necessary to obtain the required fine azimuth coregistration accuracy. Other unique aspects of processing IW data, such as azimuth spectral filtering, image resampling, and data deramping and reramping, are reviewed, giving a recipe-like description that enables the user community to use S-1 IW mode repeat-pass SAR data. Interferometric results from $S-1 A$ are provided, demonstrating the mapping capacity of the $\mathrm{S}-1$ system and its interferometric suitability for geophysical applications. An interferometric evaluation of a coherent interferometric pair over Salar de Uyuni, Bolivia, is provided, where several aspects related to coregistration, deramping, and synchronization are analyzed. Additionally, a spatiotemporal evaluation of the along-track shifts, which are directly related to the orbital/instrument timing error, measured from the SAR data is shown, which justifies the necessity to refine the azimuth shifts with ESD. The spatial evaluation indicates high stability of the azimuth shifts for several slices of a datatake.
\end{abstract}

Index Terms-Coregistration, Interferometric SAR (InSAR), Sentinel-1 (S-1), synthetic aperture radar (SAR), Terrain Observation by Progressive Scans (TOPS).

Manuscript received April 24, 2015; revised October 2, 2015; accepted October 7, 2015. Date of publication January 22, 2016; date of current version March 9, 2016. This work was supported in part by the European Space Agency under Contract 4000111074/14/NL/MP/lf, Contract 4000109669/13/ I-AM, Contract 4000106082/12/NL/MP, and Contract 4000110587/14/I-BG and in part by the German Federal Ministry for Economics and Technology through the TanDEM-X Project under Förderkennzeichen 50 EE 1035.

N. Yague-Martinez was with the Remote Sensing Technology Institute, German Aerospace Center, 82234 Weßling, Germany. He is now with the Microwaves and Radar Institute, German Aerospace Center, 82234 Weßling, Germany (e-mail: nestor.yague@dlr.de).

P. Prats-Iraola is with the Microwaves and Radar Institute, German Aerospace Center, 82234 Weßling, Germany (e-mail: pau.prats@ dlr.de).

F. Rodriguez Gonzalez, R. Brcic, R. Shau, M. Eineder, and R. Bamler are with the Remote Sensing Technology Institute, German Aerospace Center, 82234 Weßling, Germany (e-mail: Fernando.rodriguezgonzalez@dlr.de; ramon.brcic@dlr.de; robert.shau@dlr.de; michael.eineder@dlr.de; richard. bamler@dlr.de).

D. Geudtner is with the European Space and Technology Center, European Space Agency, 2201 AZ Noordwijk, The Netherlands (e-mail: dirk.geudtner@ esa.int).

Color versions of one or more of the figures in this paper are available online at http://ieeexplore.ieee.org.

Digital Object Identifier 10.1109/TGRS.2015.2497902

\section{INTRODUCTION}

$\mathbf{T}$ HE Sentinel-1 (S-1) mission is based on a constellation of identical C-band synthetic aperture radar (SAR) satellites, which are currently comprised of the A and B units, to provide data continuity to European Space Agency's (ESA) previous European Remote Sensing (ERS) and ENVISAT SAR missions.

The joint operation of both satellites will provide data sets for Copernicus Services [1] for the following areas: monitoring of sea ice zones and the arctic environment, surveillance of the marine environment, monitoring of land surface motion risks, mapping of land surfaces (forest, water, and soil), and mapping in support of humanitarian aid in crisis situations.

The S-1A unit was launched in April 2014, reaching its reference orbit on August 7, 2014. A second satellite (B unit) is scheduled for 2016 .

The S-1 system was conceived to provide repeat-pass interferometric capabilities with unprecedented wide area coverage for medium-resolution applications [2]. The repeat cycle has been notably reduced from 35 days for ERS- 1 and ERS-2 or 30/35 days for ENVISAT to 12 days for S-1A, and can still be reduced effectively to 6 days when both units are in space. The systematic data acquisition along with the exceptional temporal sampling allows a vast range of geophysical applications, such as the monitoring of cryosphere dynamics and the mapping of surface deformation, e.g., caused by tectonic processes, volcanic activities, landslides, and ground subsidence. In addition, the S-1 orbit maintenance strategy ensures a ground-track repeatability of $120 \mathrm{~m}$ resulting in small orbital InSAR baselines on the order of $150 \mathrm{~m}$. The wide-swath coverage is achieved by employing the novel Terrain Observation by Progressive Scans (TOPS) [3] acquisition mode, which similarly to ScanSAR acquires images by recording subsets of echoes of the SAR aperture, which are called bursts.

The SAR instrument, operating at $5.405 \mathrm{GHz}$, supports four imaging modes providing different resolution and coverage: Interferometric Wide Swath Mode (IW), Extra Wide Swath Mode (EW), StripMap (SM), and Wave (WV). IW and EW modes are implemented as three and five subswath TOPS SAR modes, respectively. This is to provide large swath widths of 250 and $400 \mathrm{~km}$ at ground resolutions of $5 \mathrm{~m} \times 20 \mathrm{~m}$ and $20 \mathrm{~m} \times 40 \mathrm{~m}$, respectively, with enhanced image performance as compared with the conventional ScanSAR mode [4]. The IW TOPS mode is the main mode of operations for the systematic monitoring of large land and coastal areas [2] and is the mode we will focus in this paper.

The original publication describing the TOPS principle by De Zan and Monti Guarnieri [3] covers all aspects of this 
new SAR mode, including the requirements for interferometry. Further aspects were considered in [5]. The TOPS mode was first implemented as an experimental four-subswath mode on TerraSAR-X [6]-[8]. The feasibility of repeat-pass TOPS SAR for interferometry was demonstrated in [9] for stationary scenes and in [10] for nonstationary surface scenarios, such as glaciers.

Prior to the launch of S-1A, an experimental three-subswath TOPS mode was implemented on RADARSAT-2, operating at the same C-band frequency as S-1, to simulate S-1-like IW mode data products to support the development of processing and exploitation of S-1 IW TOPS data with a particular focus on SAR interferometry [11], [12].

Other publications concerned with efficient focusing of TOPS acquisitions have appeared in the last years [13]-[16], including experiments with an airborne SAR in [17].

In [18], the description of burst-mode interferometric signal properties is provided, covering, among other issues, azimuth scanning pattern synchronization, spectral shift filtering in the presence of high squint coregistration and subswath alignment, and ScanSAR interferogram formation.

This paper focuses on the interferometric processing of S-1 IW mode data acquired over stationary scenes and provides a recipe-like description of the required operations. We summarize the experience and results obtained with two independent InSAR processors developed at the DLR, which are the Integrated Wide Area Processor (IWAP) [19] and the experimental TanDEM-X interferometric processor (TAXI) [20]. In order to illustrate some important parameters and intermediate results of IW data, we have selected an S-1A IW mode InSAR data pair acquired over Salar de Uyuni, Bolivia.

This paper is structured as follows. Section II provides a description of the S-1 IW mode, including the burst spectral properties and the key parameters of the single-look complex (SLC) data product. Moreover, based on the IW mode burst spectral properties, we discuss the impact of burst missynchronization on azimuth spectral alignment and evaluate the required accuracy for azimuth coregistration of S-1A IW burst images. Section III provides a recipe for interferometric processing, covering coregistration, spectral shift filtering and consideration of the Doppler frequency variation. In Section IV S-1A interferometric results are provided. Finally, Section V presents an interferometric evaluation performed with S-1A data by using a stack of acquisitions over Mexico City. The along-track shifts are systematically analyzed using two types of orbits. In addition, the common Doppler bandwidth is evaluated. A spatial analysis of the along-track shift is provided using a datatake over Europe. In the Appendix, we briefly review the S-1 data product description and outline practical information on how to handle the IW SLC data format.

\section{S-1 IW ModE}

The TOPS SAR acquisition mode is capable of providing wide range swaths as with the ScanSAR technique, but it almost eliminates the associated problems of scalloping and azimuth varying signal-to-noise ratio, noise equivalent sigma zero, and azimuth ambiguities [3].

In addition to scanning in elevation in order to extend the range coverage, the antenna azimuth beam is steered electron-

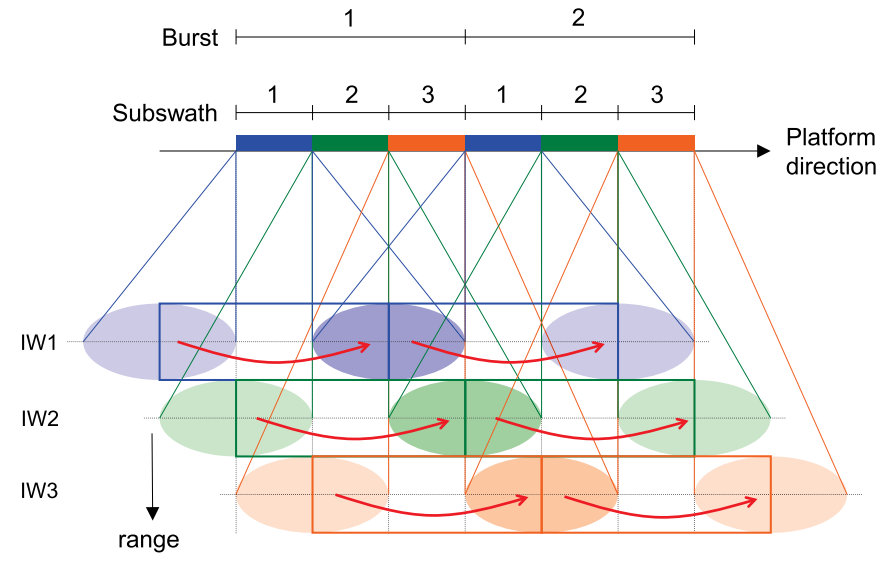

Fig. 1. TOPS Scan pattern for S-1 IW mode, composed of three subswaths. The acquisition starts with the first burst of the first subswath (blue) at top left with the beam steered along azimuth in the same direction as the platform moves (as depicted by the red arrows). Once this burst has been acquired, the antenna is switched in elevation, and the first burst of the second subswath (green) is acquired. Once the first of the third subswath (orange) is acquired, the beam is switched back to the first subswath, and the process is cyclically repeated.

ically from aft to fore at a constant rate. The scan pattern is shown in Fig. 1. As a result and in contrary to ScanSAR, all targets on the ground are observed by the entire azimuth antenna pattern. The acquisition takes place by recording bursts of echoes, i.e., employing subapertures, at the expense of a lower azimuth resolution. S-1 employs three predefined subswaths in IW mode (IW1, IW2, and IW3), achieving a ground swath coverage of $250 \mathrm{~km}$ in the across-track direction.

$\mathrm{S}-1 \mathrm{~A}$ is capable of operating up to $25 \mathrm{~min}$ per orbit [21]; thus, a slicing procedure has been defined for the generation of products. Regarding the L1 SLC products, each product slice is provided as a segment of approximately $25 \mathrm{~s}$ in length, which corresponds to about $170 \mathrm{~km}$. An overlap area of about 7\%-8\% is present in the azimuth direction between consecutive focused bursts, as well as in range between adjacent subswaths. This assures that the images can be mosaicked without any gap. Moreover, as will be shown in Section III-D, these areas will be exploited to obtain the necessary coregistration accuracy for interferometric processing.

Table I lists the most relevant SLC product parameters of S-1 IW mode. The indicated incidence angles are approximate since a roll steering law is applied to the spacecraft to compensate for altitude variations [4].

\section{A. Spectral Properties}

The nonstationarity of the squint angle during the TOPS acquisition produces a linear variation of the Doppler centroid frequency in the SAR data. Fig. 2 shows the (unfolded) spectrum of a single raw burst of duration $T_{\text {burst }}$. Observe that the azimuth resolution is controlled by the dwell time $T_{\text {dwell }}$ (integration time for a point target), resulting in a target bandwidth $B_{T}=\left|k_{a}\right| \cdot T_{\mathrm{dwell}}$, with $k_{a}$ being the Doppler rate. The Doppler rate introduced by the antenna steering is given by

$$
k_{\mathrm{rot}} \approx \frac{2 v}{\lambda} \omega_{r}
$$


TABLE I

S1 INTERFEROMETRIC WIDE SWATH MODE SLC PRODUCT PARAMETERS

\begin{tabular}{|c|c|c|c|}
\hline Beam id. & IW1 & IW2 & IW3 \\
\hline Incidence angles & $32.9^{\circ}$ & $38.3^{\circ}$ & $43.1^{\circ}$ \\
\hline Slant range resolution & $2.7 \mathrm{~m}$ & $3.1 \mathrm{~m}$ & $3.5 \mathrm{~m}$ \\
\hline Range Bandwith & $56.5 \mathrm{MHz}$ & $48.3 \mathrm{MHz}$ & $42.79 \mathrm{MHz}$ \\
\hline Azimuth resolution & $22.5 \mathrm{~m}$ & $22.7 \mathrm{~m}$ & $22.6 \mathrm{~m}$ \\
\hline Processing Bandwidth & $327 \mathrm{~Hz}$ & $313 \mathrm{~Hz}$ & $314 \mathrm{~Hz}$ \\
\hline Doppler Centroid span $\left(\Delta f_{\mathrm{DC}}\right)$ & $5.2 \mathrm{kHz}$ & $4.4 \mathrm{kHz}$ & $4.6 \mathrm{kHz}$ \\
\hline Slant range pixel spacing & \multicolumn{3}{|c|}{$2.3 \mathrm{~m}$} \\
\hline Range sampling frequency & \multicolumn{3}{|c|}{$64.35 \mathrm{MHz}$} \\
\hline Azimuth pixel spacing & \multicolumn{3}{|c|}{$14.1 \mathrm{~m}$} \\
\hline Azimuth sampling frequency & \multicolumn{3}{|c|}{$486.49 \mathrm{~Hz}$} \\
\hline Azimuth steering angle & \multicolumn{3}{|c|}{ $\pm 0.6^{\circ}$} \\
\hline Burst length ( $\left.T_{\text {focused }}\right)$ & \multicolumn{3}{|c|}{$2.75 \mathrm{~s} / \approx 20 \mathrm{~km}$} \\
\hline Ground Swath width & \multicolumn{3}{|c|}{$250 \mathrm{~km}$} \\
\hline Slice length & \multicolumn{3}{|c|}{$170 \mathrm{~km}$} \\
\hline Orbital Repeat Cycle & \multicolumn{3}{|c|}{12 days } \\
\hline Orbit height & \multicolumn{3}{|c|}{$698-726 \mathrm{~km}$} \\
\hline Wavelength & \multicolumn{3}{|c|}{$5.547 \mathrm{~cm}$} \\
\hline Polarization & \multicolumn{3}{|c|}{$\begin{array}{c}\text { Single }(\mathrm{HH} \text { or } \mathrm{VV}) \text { or } \\
\text { Dual }(\mathrm{HH}+\mathrm{HV} \text { or } \mathrm{VV}+\mathrm{VH})\end{array}$} \\
\hline
\end{tabular}

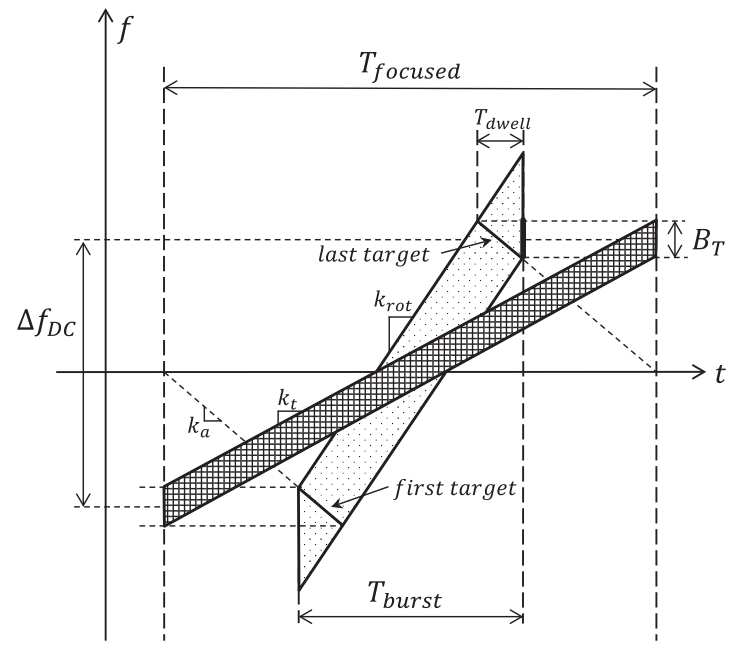

Fig. 2. Time-frequency diagram of a single TOPS burst. The raw burst (dotted pattern) of duration $T_{\text {burst }}$ presents a linear Doppler variation due to the steering of the antenna. The zero-Doppler focused burst (hashed pattern) of duration $T_{\text {focused }}$ also exhibits a linear Doppler variation. The first and last observed target Doppler histories are depicted with bold lines and have a slope given by the Doppler rate $k_{a}$. Their zero-crossing positions determine the length of the focused burst. The dwell time $T_{\text {dwell }}$ controls the azimuth resolution, being the resulting target bandwidth $B_{T}$.

where $v$ is the satellite velocity, $\lambda$ is the radar wavelength, and $\omega_{r}$ is the antenna steering rate in radians per second.

After the burst raw data have been focused to SLC bursts in zero-Doppler geometry, a linear Doppler centroid frequency variation is present in the azimuth direction. The range-dependent Doppler rate at image level $k_{t}(r)$ can be calculated as

$$
k_{t}(r)=\frac{k_{a}(r) \cdot k_{\mathrm{rot}}}{k_{a}(r)-k_{\mathrm{rot}}}
$$

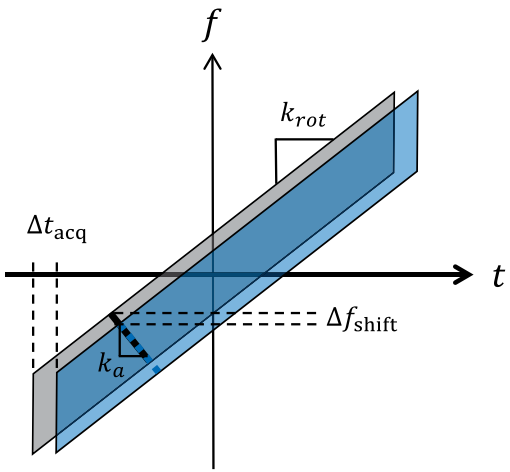

Fig. 3. Time-frequency diagram of a pair of raw bursts depicting the azimuth spectra in the presence of a timing error $\Delta t_{\text {acq }}$. The master burst is depicted in gray and the slave in blue. The plot depicts the case where the master datatake observes the target before the slave datatake. Note that the delay of observation of each target is shorter than the delay of the overall acquisition.

where $k_{a}(r)$ is the range-dependent target Doppler rate.

This Doppler variation has to be considered for further InSAR processing steps, including interpolation and spectral shift filtering, as will be discussed in Section III-B.

\section{B. Burst Synchronization Aspects for Interferometry}

Burst synchronization relates to the fact that the satellite must be at the same along-track position for both passes in order to observe the targets with the same squint angle. A lack of spectral overlap due to burst mis-synchronization leads to decorrelation. The need for burst synchronization puts requirements on commanding, namely on the accuracy of data acquisition timing, as depicted in Fig. 3. The Doppler shift is given by

$$
\Delta f_{\text {shift }}(r)=k_{t}(r) \cdot \Delta t_{\text {acq }}
$$

where $\Delta t_{\text {acq }}$ is the timing difference between both acquisitions (raw data), and $k_{t}$ is the Doppler rate at image level, which is given by (2).

However, not only the accuracy of the synchronization at the beginning of the datatakes is relevant, the size of the orbital tube in the radial dimension affects synchronization during the datatake, i.e., $\Delta t_{\mathrm{acq}}$ varies during the acquisition. This effect has been addressed in [22] in the frame of the S-1 mission.

The coherence loss can be avoided if proper azimuth spectral shift filtering is performed, at the cost of a reduced azimuth resolution. The calculation of the timing difference between both acquisitions and the final common Doppler bandwidth will be provided in Section III-E.

\section{Coregistration Accuracy}

For conventional nonsquinted stripmap acquisitions, misregistration does not introduce a phase bias but may increase the phase variance [23]. An accuracy of 0.1 pixels is usually sufficient to obtain high-quality interferograms. The coregistration requirements for TOPS mode are however much more demanding due to the significant Doppler frequency variation. In [24] and [25], an analysis of the phase error introduced by a 
misregistration in the case of squinted acquisitions shows that the variation in squint angle over azimuth and, to a lesser extent, over range cause phase ramps in both azimuth and range if a misregistration is present. We focus on the azimuth misregistration error since the greater variation of the squint angle in this direction produces much more pronounced phase ramps in azimuth. Using a small-angle approximation, a misregistration of $\Delta t$ seconds in azimuth between master and slave leads to an azimuth phase ramp over the burst of

$$
\Delta \phi_{\mathrm{az}}=2 \pi \Delta f_{\mathrm{DC}} \Delta t=2 \pi \frac{2 v}{\lambda} \Delta \beta \Delta t[\mathrm{rad}]
$$

where $\Delta f_{\mathrm{DC}}$ is the change in Doppler centroid over azimuth, $\Delta \beta$ is the corresponding change in squint angle, and $\Delta t$ is the misregistration.

Considering the maximum $\Delta f_{\mathrm{DC}}$ of $5.2 \mathrm{kHz}$, in order to limit the phase ramp to, for example, $1 / 100$ cycle $\left(=3.6^{\circ}\right)$, coregistration accuracy of approximately 0.0009 pixels at an azimuth sampling frequency $f_{\text {az }}$ of $486 \mathrm{~Hz}$ would be required, which is equivalent to circa $1.9 \mu \mathrm{s}$ or $1.3 \mathrm{~cm}$.

The high accuracy requirement for the azimuth coregistration limits on the use of traditional methods as, for example, crosscorrelation with small patches distributed over the scene, where each correlation estimate is taken directly as the shift for the center position of the patch. Section III-D addresses the coregistration approach.

\section{INSAR PROCESSING FlOW}

Here, the methodology for interferometric processing is provided. Some details on the importing of the SLC data are first discussed, and the processing chain at burst level is presented. The remaining part concentrates on specific algorithms, namely, deramping the focused bursts, coregistration, spectral shift filtering, and mosaicking of the bursts.

The data can be handled following a three-level hierarchical structure. The lowest level is the burst-level, at which interferometric processing is performed, e.g., signal interpolation and spectral shift filtering. The next higher level is the subswath where mosaics of the bursts are generated. The highest level corresponds to the slice, at which a mosaicking of all three subswaths can be performed.

The philosophy that we have adopted for the coregistration consists of performing a geometric coregistration using an external digital elevation model (DEM) and orbit information, followed by a correction of the residual shift in range and azimuth estimated from the SAR data. This residual shift can be due to orbit inaccuracies, timing errors, or physical effects (e.g., troposphere or solid Earth tides).

\section{A. Data Importing and Preparation}

The processing starts with the importing of the master and slave SLC data products; some details on the format of the products can be found in Appendix. The L1 SLC data are generated by the operational ESA Instrument Processing Facility (IPF). It is important to emphasize that all interferometric operations, e.g., spectral shift filtering or burst resampling, have to be done at burst-level, in order to properly consider the Doppler centroid frequency variation. Thus, the data reader should be able to extract the single SLC bursts with their associated annotation. Although not necessary if nominal slice framing has been applied by the IPF, it is good practice to find the corresponding master and slave burst-pairs. It is sufficient to perform this calculation once per subswath. The possible azimuth wholeburst offset can be retrieved by performing a geolocation of an arbitrary slant-range point included in each subswath, e.g., midpoint, using the master orbit to obtain the position on ground. Afterward, an inverse geolocation of this point using the slave orbit provides the slant-range coordinates for the slave point. With the azimuth burst length and the subswath timing information, the whole-burst offset can be easily obtained.

\section{B. Deramping Function}

The consideration of the Doppler centroid frequency of the focused burst is critical for interferometric processing. In [26], the procedure for calculating the deramping function for S-1 products is provided and will not be repeated here.

There are two possible approaches to correctly account for the Doppler centroid when performing interpolation and/or filtering operations on the bursts.

1) Demodulate data: The complex data are deramped in azimuth in order to obtain a low-pass signal. This approach is appropriate for spectral shift filtering and for resampling. The data have to be reramped after the filtering/ resampling has been performed, where in the case of the resampling, one needs to resample also the reramping function before applying it to the data. Note that the deramping and the demodulation introduce a range spectral shift due to their slight range dependence. However, this shift can be neglected for the S-1 case.

2) Modulate kernel: The data are not demodulated, but the kernel is modulated in azimuth such that its spectrum follows the local Doppler centroid of the data. This approach can be more convenient when resampling the complex bursts and avoids the separate resampling of the complex data and the deramping function as in the previous case. However, one needs to consider the Doppler variation within the kernel's length to avoid aliasing. For S-1, this variation is about $4 \mathrm{~Hz} / \mathrm{sample}$, which, given the oversampling of the azimuth signal of $160 \mathrm{~Hz}$ (approximately $32 \%$ ), does not impose a critical kernel length.

\section{Burst-Level Interferometric Processing}

Fig. 4 shows a simplified block diagram of the interferometric processing of every burst. The Enhanced Spectral Diversity (ESD) technique [9] is applied for the retrieval of the fine (rigid) azimuth shift. In the first iteration, a geometric coregistration is performed to ensure negligible coherence loss for interferogram formation and the calculation of the subsequent differential interferogram in the overlap area. An external DEM and precise orbit information is used for this coarse coregistration. Interpolation of the slave bursts is performed with a six-point cubic convolution kernel [27]. For each azimuth position, as 


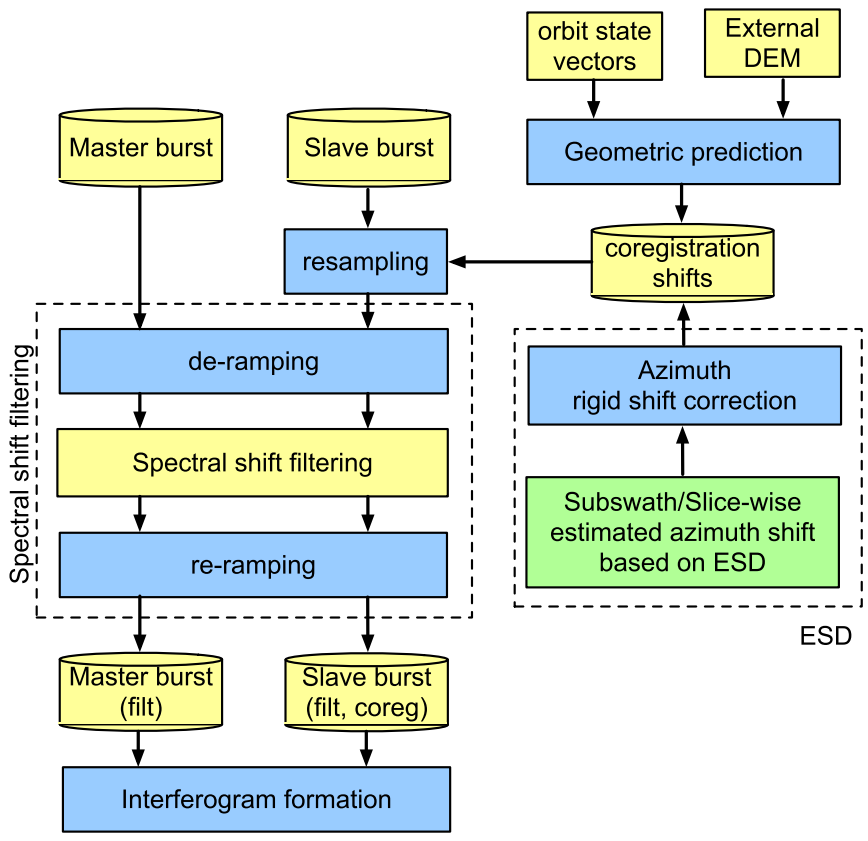

Fig. 4. Burst-level interferometric processing. Resampling, spectral shift filtering, in range and azimuth, and interferogram formation are computed at this level. A geometric coregistration is performed, which is corrected by using ESD.

with Spotlight data, the kernel is modulated in azimuth with a linear phase function so that its spectrum is centered at the local Doppler centroid frequency of the data. Spectral shift filtering is also performed in order to increase the coherence. The master and slave bursts are filtered to a common range and azimuth bandwidth. ESD can be applied by calculating the differential interferogram on the overlap areas between consecutive bursts (along the subswath). Section III-D will give more details on the estimation of the azimuth shift. Once the rigid shift has been retrieved, several possibilities arise for the second iteration. A first option is to repeat the procedure by correcting the coregistration shifts, resampling the slave, and filtering master and slave bursts to a common bandwidth. A second more efficient option is to apply the fine azimuth shift to the slave burst by multiplying its Doppler Spectrum by a linear phase term (not depicted in the figure).

\section{Coregistration}

First, a geometric coregistration, using an external DEM and orbit information, is performed. This state-of-the-art procedure is precisely described in [28] and not detailed here. There are however some interesting aspects to consider regarding the accuracy of the external DEM for the geometric azimuth coregistration in case of squinted acquisitions, which are covered in [22].

It is important to note that there are three orbit products available: the orbit information annotated in the SLC product, the restituted orbit, and the precise orbit. The last two are provided by ESA as independent products. The restituted orbit is available just a few hours after the acquisition and establishes an accuracy requirement of $10 \mathrm{~cm} 2-\mathrm{D} 1$-sigma, where 2-D means the along-track and cross-track directions. The precise orbit product, available 20 days after the acquisition, establishes an accuracy requirement of $5 \mathrm{~cm} 3-\mathrm{D}$ 1-sigma [29].

From our experience, we do not recommend the use of the orbit information annotated in the SLC product for accurate calculations. However, ESA has confirmed that this issue is currently being addressed as enhanced orbit accuracy of the annotated orbit can be expected in the future.

The geometric coregistration is followed by a refinement of the shifts exploiting the SAR data. Regarding the range direction, cross-correlation can be applied to patches distributed over the master and slave SLCs and a linear correction of the shifts can be performed.

Regarding the azimuth direction, it is important to distinguish between two possible scenarios:

1) Stationary: No deformation is expected, or if present, there is no component in the azimuth direction. The azimuth correction to apply is, in essence, a rigid shift due to a possible timing error.

2) Nonstationary: The phase jumps that may appear at the interface between adjacent bursts in the case of ground deformation in the azimuth direction have already been addressed in [30]. The reason for the phase jumps is the different projection of the azimuth shift onto the (changing) line of sight. In [30], it is proposed not to perform a local azimuth coregistration if the displacement is sufficiently small such that there is sufficient coherence but to compensate solely for the timing error. At a latter stage, the differential phase should be correctly interpreted by taking into account the actual line-of-sight for each pixel.

We will focus on the stationary case. Several possibilities arise for the retrieval of the constant azimuth shift. A first possibility is to apply cross-correlation techniques [31] - in its coherent (CCC) or incoherent (ICC) versions - to patches distributed over the master and slave SLCs and afterward average the residual azimuth shift to correct for the rigid shift, as already proposed in [32]. Another possibility is to use the ESD technique [9], which exploits the large Doppler frequency difference in the overlap areas between adjacent bursts. The main advantage of correlation techniques over ESD is that they provide a nonambiguous measurement, whereas some care has to be taken when using ESD since it exploits the phase, meaning the retrieval of the correct ambiguity band may not be possible. If the performance of ICC [33] is compared with the performance of ESD, we see that ESD outperforms ICC by approximately one order of magnitude for the S-1 parameters. Fig. 5 compares the standard deviation of both estimators when using all bursts in a single subswath. The overlap regions between bursts within the subswath are assumed used for ESD and all pixels within the subswath for ICC. The exponential decorrelation model [34] with a decorrelation time of 35 days was used to predict the coherence after 1-6 12-day repeat-pass cycles.

We have chosen the ESD technique to obtain the fine azimuth coregistration accuracy; however, it is possible that for certain scenarios (e.g., islands, where the overlap areas correspond to water and therefore incoherent, or if the ambiguity band is not solved), ICC can be useful. A combination of both 


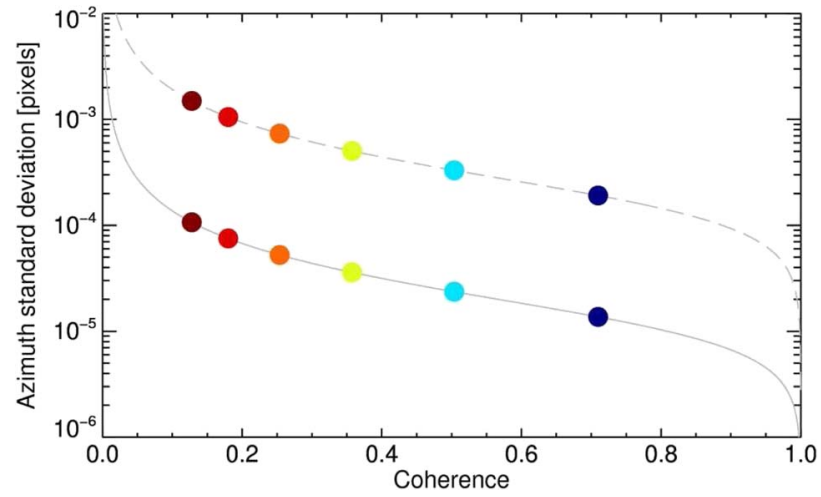

Fig. 5. Azimuth coregistration accuracy for IW2 using ICC (dashed) and ESD (solid). The circles (blue to red) correspond to the coherence after 1-6 12-day repeat-pass cycles, respectively, using the exponential decorrelation model with a decorrelation time of 35 days.

TABLE II

ESD AMBiguity BAND AND Three-Sigma (99.73\%) VALues of THE EXPECTED ALONG-TRACK ACCURACY WHEN COMBINING Two (MASTER AND SLAVE ACQuisitions) Precise OR RESTITUTED ORBITS

\begin{tabular}{|c|c|c|c|}
\hline & IW1 & IW2 & IW3 \\
\hline$\left\langle\Delta f_{\mathrm{DC}}^{\text {ovl }}\right\rangle[\mathrm{Hz}]$ & 4814.25 & 4044.80 & 4267.22 \\
\hline Ambiguity band [pix] & \pm 0.051 & \pm 0.060 & \pm 0.057 \\
\hline Ambiguity band [m] & \pm 0.71 & \pm 0.85 & \pm 0.80 \\
\hline Precise Orbit $3 \sigma_{1 \mathrm{D}}^{\text {InSAR }}[\mathrm{m}]$ & \multicolumn{3}{|c|}{0.12} \\
\hline Restituted Orbit $3 \sigma_{1 \mathrm{D}}^{\text {InSAR }}[\mathrm{m}]$ & \multicolumn{3}{|c}{0.30} \\
\hline
\end{tabular}

methods is also possible, where ICC is employed to determine the ambiguity band, applying afterward ESD to obtain a fine estimate of the shift. The approximate main ambiguity band is detailed in Table II for each subswath. The three-sigma values of the expected along-track accuracy when combining two (master and slave acquisitions) precise or restituted orbits are also provided, indicating that the ambiguity band is already solved if the geometric coregistration has been performed with precise or restituted orbits. An isotropic distribution of the error in the different components (along-track and cross-track for the restituted orbit and radial, and along-track and cross-track for the precise orbit), has been assumed for the calculation of the expected along-track accuracy, which, although generally not true, is sufficient to obtain an order of magnitude estimate of the expected accuracy. A systematic evaluation of the azimuth shifts with S-1A data in Section V confirms this.

ESD exploits the phase difference in the overlap area between adjacent bursts within a subswath and between adjacent bursts from subswath-to-subswath. We will focus our analysis on the exploitation of the overlap areas within a subswath. The overlap areas from subswath-to-subswath can also be employed, as proposed in [35]. Using these overlap areas improves the performance of the estimation and can also help to resolve the ambiguity band since the Doppler differences in these areas are smaller than in the overlap areas within each subswath, resulting in a larger ambiguity band.

The ESD phase can be calculated for every pixel of each overlap area as follows:

$$
\phi_{\mathrm{ESD}}=\arg \left\{\left(m_{i} \cdot s_{i}^{*}\right) \cdot\left(m_{i+1} \cdot s_{i+1}^{*}\right)^{*}\right\}
$$

where $m_{i}$ and $s_{i}$ refer to the $i$ th master and slave complex bursts, respectively; $m_{i+1}$ and $s_{i+1}$ refer to the $(i+1)$ th master and slave bursts, respectively; and $\arg \{\cdot\}$ gives the phase of a complex number.

Rewriting (4), an azimuth coregistration error of $\Delta y$ pixels for pixel $p$ causes an interferometric phase difference, in radians, of [9], [25]

$$
\phi_{\mathrm{ESD}, p}=2 \pi \Delta f_{\mathrm{DC}, p}^{\mathrm{ovl}} \frac{\Delta y}{f_{\mathrm{az}}}
$$

where $\Delta f_{\mathrm{DC}, p}^{\mathrm{ovl}}$ is the Doppler centroid frequency difference in the overlap area for each burst, and $f_{\mathrm{az}}$ is the image azimuth sampling frequency.

A first approximation for the estimation of the shift would be to average the ESD phase and the Doppler centroid frequency differences, i.e.,

$$
\widehat{\Delta y}=\frac{f_{\mathrm{az}}}{2 \pi} \cdot \frac{\arg \left\{\left\langle e^{j \phi_{\mathrm{ESD}, p}}\right\rangle\right\}}{\left\langle\Delta f_{\mathrm{DC}, p}^{\text {ovl }}\right\rangle}
$$

where $\langle\cdot\rangle$ indicates average value.

We propose to model the ESD phase at each pixel, induced by a constant azimuth shift within the overlap area, using the local Doppler centroid frequency difference.

The estimation of the azimuth coregistration shift $\Delta y$ requires accounting not only for the local Doppler centroid frequency differences but also for the wrapped nature of the differential phase, $\phi_{\mathrm{ESD}, p}$. It is not possible to divide the phase difference values by the local shift-to-phase conversion factor according to (6); otherwise, the estimation would be biased.

The following estimator is proposed:

$$
\widehat{\Delta y}=\underset{\Delta y}{\arg \min }\left\{\left|\arg \sum_{p} e^{j\left(\phi_{\mathrm{ESD}, p}-2 \pi \Delta f_{\mathrm{DC}, p}^{\mathrm{ov}}, \frac{\Delta y}{f_{\mathrm{az}}}\right)}\right|\right\} .
$$

Another approach consists of maximizing the absolute value of the real part, as proposed in [36]. A weighted estimation using the coherence can also be considered, but this is not included in (8) for simplicity.

Its application is only valid for shifts smaller than the smallest of the ambiguity bands of the overlap areas used within the estimation. Thus, if multiple bands of ambiguity are present, as in the case of a combination of the overlap areas within one subswath but for all swaths jointly (IW1-IW1, IW2-IW2, and IW3-IW3), then the search should be restricted to the ambiguity band of IW1.

The value of the estimator for different shift values and the residual phase after compensation of the phase created by the estimated shift is depicted in Fig. 6. The parameters have been calculated for S-1A data in IW mode over Salar de Uyuni. Due to the small variation of the Doppler difference, the estimator is quasi-cyclic. Thus, the search space must be constrained to one ambiguity band according to (6).

As introduced in [37], some spatial multilooking of the interferograms in the overlap area prior to calculation of the differential interferogram (so-called early-multilooking) increases the estimation accuracy. The effect on the histogram of the residual differential phase for a scene-wise estimation (i.e., considering all overlap areas within each subswath) is depicted 
(a) Phase difference

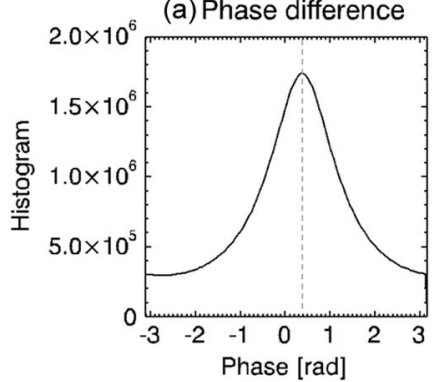

(c) Residual

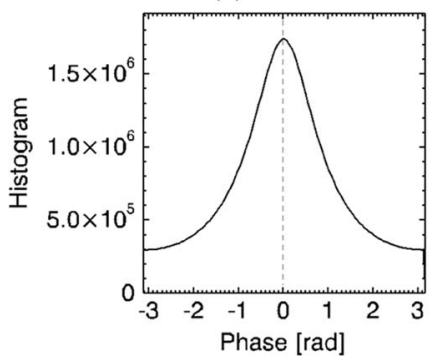

(b) Estimation

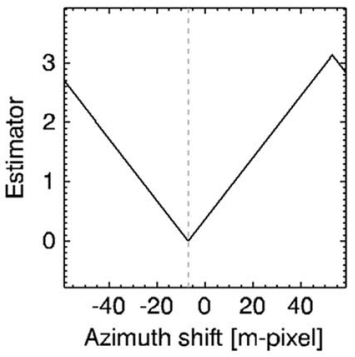

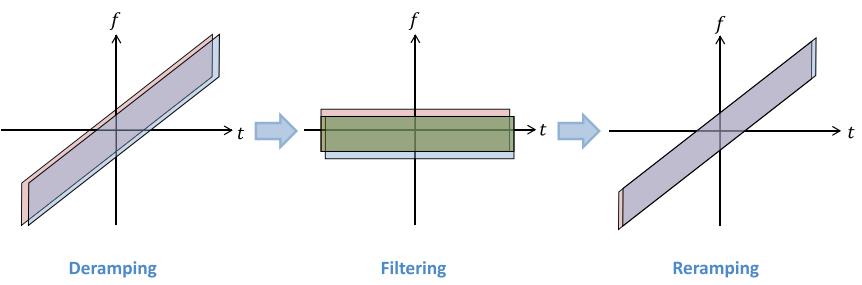

Fig. 8. Time-frequency diagrams at image level showing the rationale of the azimuth spectral filtering process. In the example, only the burst missynchronization is depicted, i.e., it is assumed that the Doppler centroid for both datatakes is $0 \mathrm{~Hz}$ and that there is no contribution due to the crossing orbits. The master is depicted in orange and the slave in blue. (Left) Original signals. (middle) After deramping with the master deramping function, where it can be observed that the burst mis-synchronization introduces spectral decorrelation. The green block represents the bandpass spectral filter. (Right) After reramping.

bandwidth. Conventional range spectral filtering can be applied in TOPS mode as usual, but filtering in azimuth requires special care due to the time-variant Doppler change over azimuth. The principle of azimuth spectral filtering is the same as for Spotlight interferometry, i.e., deramping and reramping operations must be performed prior to and after filtering, as described in detail in [39].

The calculation of the common Doppler bandwidth for each pixel $p$ due to possible Doppler centroid differences and to the burst mis-synchronization can be calculated as

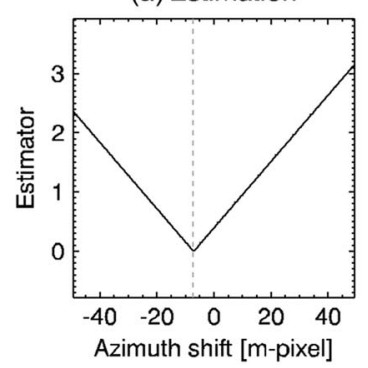

(c) Estimation

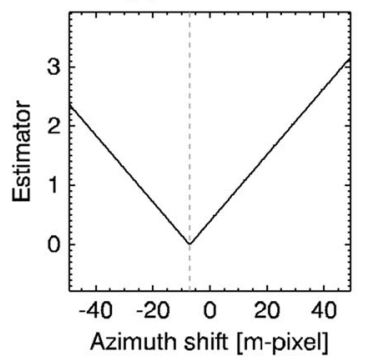

(b) Residual

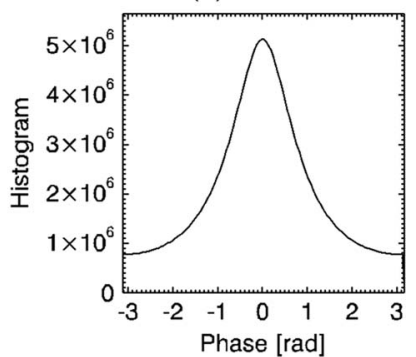

(d) Residual

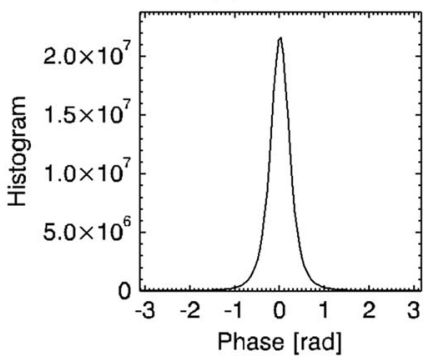

Fig. 7. Effect of burst multilooking in azimuth offset estimation. (a) and (b) Estimation and residual phase without burst multilooking. (c) and (d) Estimation and residual phase with burst multilooking (25 looks). The plots are derived from the S-1A interferometric pair over Salar de Uyuni.

in Fig. 7. (a) and (b) show the estimation result and the residual differential phase histogram if no-multilooking is performed, whereas (c) and (d) show the same results if a multilooking of 25 looks has been applied to the interferograms.

\section{E. Spectral Shift Filtering}

The different acquisition geometries for an interferometric pair introduce spectral decorrelation [38]. This effect can be avoided by filtering the master and slave bursts to a common

$B_{\text {common }, p}=B_{a}-\left|f_{\mathrm{DC}, p}^{\text {master }}-\left(f_{\mathrm{DC}, p}^{\text {slave_resampled }}+\Delta f_{\text {orbit }}\right)\right|$

where $B_{a}$ is the azimuth bandwidth, $\Delta f_{\text {orbit }}$ is the spectral shift due to the nonparallel orbits, $f_{\mathrm{DC}, p}^{\text {master }}$ is the Doppler centroid of the master burst for each pixel, and $f_{\mathrm{DC}, p}^{\text {slaveresampled }}$ is the Doppler centroid of the slave burst mapped to the master burst geometry. The rationale of azimuth spectral filtering is depicted in Fig. 8.

As for all the steps in burst interferometric processing, the parameters must be independently determined for each of the bursts. Fig. 9(a), (c), and (e) shows the Doppler centroid frequency over range of IW1, IW2, and IW3 for an interferometric pair over Salar de Uyuni after a deramping has been applied. Note that the Doppler centroid frequencies are close to zero for both channels, the difference in Doppler frequency being only a few Hertz. The Doppler centroid frequency rate varies considerably over range, as shown in Fig. 9(b), (d), and (f) for IW1, IW2, and IW3, respectively. As a consequence, the deramping and reramping operators must be adapted to the local Doppler centroid rate for each range bin.

The range spectral filtering does not require an update for TOPS interferometry. Fig. 10 displays the range fringe frequency considering flat Earth for all three subswaths. The plots show the local fringe frequency and a constant fringe frequency calculated at mid burst. As it can be observed, the local fringe frequency varies over range, being this variation more significant for subswath IW1. In any case, note that, due to the small orbital tube of S-1 (kept to a radius of less than $100 \mathrm{~m}$ ) and the large range bandwidth (given in Table I), the range spectral filter will seldom be necessary. 
(a) IW1

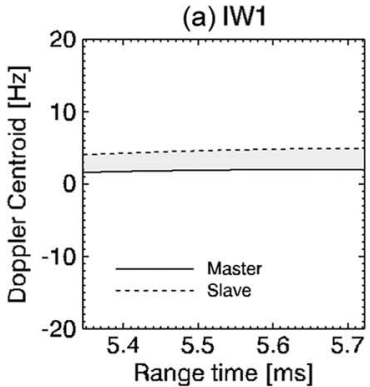

(c) IW2

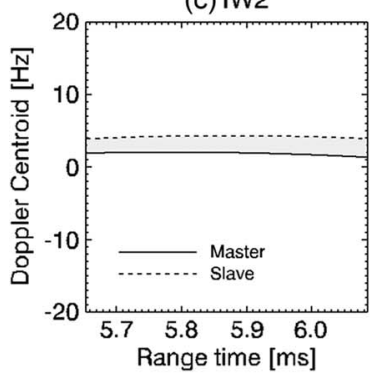

(e) IW3

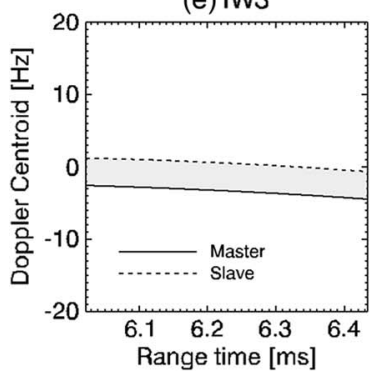

(b) IW1

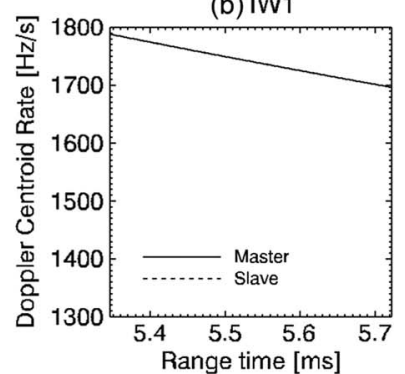

(d) IW2

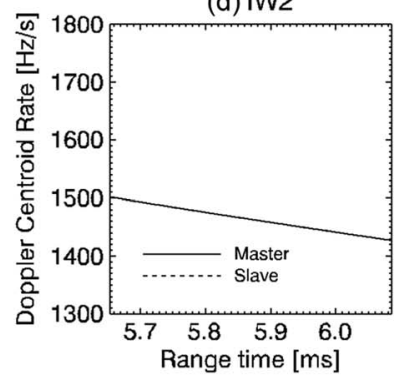

(f) IW3

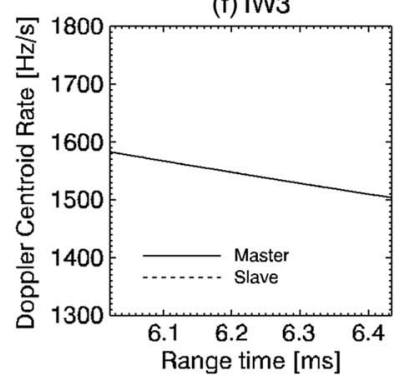

Fig. 9. Master and slave azimuth parameters for spectral shift filtering (after deramping has been applied). (a),(c), and (e) Doppler centroid frequency in range at azimuth center of burst. (b), (d), and (f) Doppler centroid frequency rate at the azimuth center of bursts. Slave parameters have been coregistered to master time. The parameters have been extracted from a S-1A interferometric pair over Salar de Uyuni.
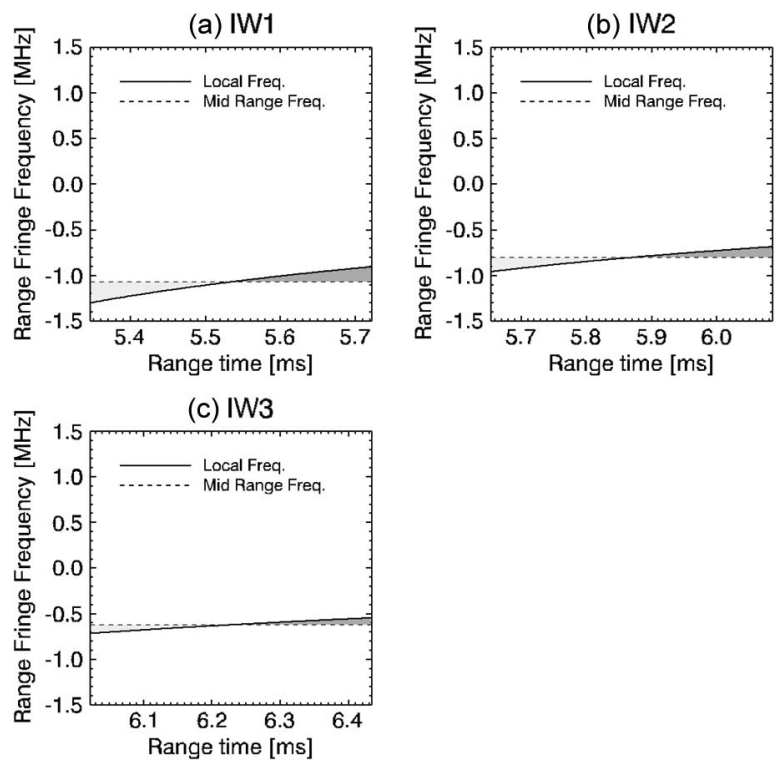

Fig. 10. Range fringe frequency (considering flat earth) for all three subswaths. The plots indicate the local fringe frequency (continuous line) and a constant fringe frequency, calculated at mid burst. The parameters have been extracted from a S-1A interferometric pair over Salar de Uyuni, which presents an effective baseline of $108 \mathrm{~m}$.

\section{F. Mosaicking of Bursts Within a Slice}

After the slave bursts have been resampled, the burst interferograms can be computed, and the coherence can be estimated. At this stage, it is important to account for the pixel validity, whose information can be extracted from the $\mathrm{L} 1$ products. The general approach is to mosaic the full-resolution interferogram (pixelwise by definition) first, followed by phase flattening and multilooking operations.

There are some minor points to consider with respect to mosaicking the bursts to produce subswath or scene-level interferograms. Three approaches to mosaicking appear possible.

1) Join at a certain azimuth or range line such as the azimuth midpoint of the overlap area between bursts within a subswath or the range midpoint of the overlap area between subswaths.

2) Loop over all bursts, with the current burst overwriting the mosaic but only when the burst pixel is valid as determined from the burst's valid pixel mask.

3) Perform a weighted average within the overlap area. This approach can quickly become complicated when considering the valid pixel masks and applying roll-off functions in both the range and azimuth directions within the overlap area to smooth the transition.

We do not recommend the third approach even if the phase quality can be enhanced by averaging both looks. By consistently cutting at fixed range and azimuth lines, it is easier to visually identify phase discontinuities between bursts due to residual misregistration, for quality analysis purposes or more interestingly in the case of scenarios with horizontal displacement in the azimuth direction.

The flat Earth phase can be subtracted once the mosaic of the interferogram has been generated and differential phase can be obtained by subtracting a DEM simulated phase. Finally, interferogram multilooking is performed.

\section{First Interferometric Results}

The first IW InSAR pair was available in ascending geometry over the Gulf of Genoa, Italy, on August 19, 2014, 12 days after S-1A had reached its final orbit on August 7, 2014. This first TOPS pair was processed some hours later, demonstrating the readiness of the S-1 system (both in-orbit instrument and on-ground commanding and processing) for interferometric applications. Fig. 11 shows the interferometric phase and SAR amplitude overlay image of the mosaic of the first two slices over Italy. The image is composed of three subswaths and has a ground range extension of $250 \mathrm{~km}$. The effective baseline is about $120 \mathrm{~m}$. After flat Earth phase removal, one fringe color cycle corresponds to a height of ambiguity of about $129 \mathrm{~m}$.

A further interferometric acquisition over Italy in ascending geometry was available from August 9 and 21. A mosaic of the interferometric phase, having removed a simulated phase from a DEM from the Shuttle Radar Topography Mission (SRTM), overlaid with the SAR amplitude (left) and coherence (right) of the datatake, is shown in Fig. 12. The acquisition extends from Sicily to the Alps demonstrating the high mapping capabilities 

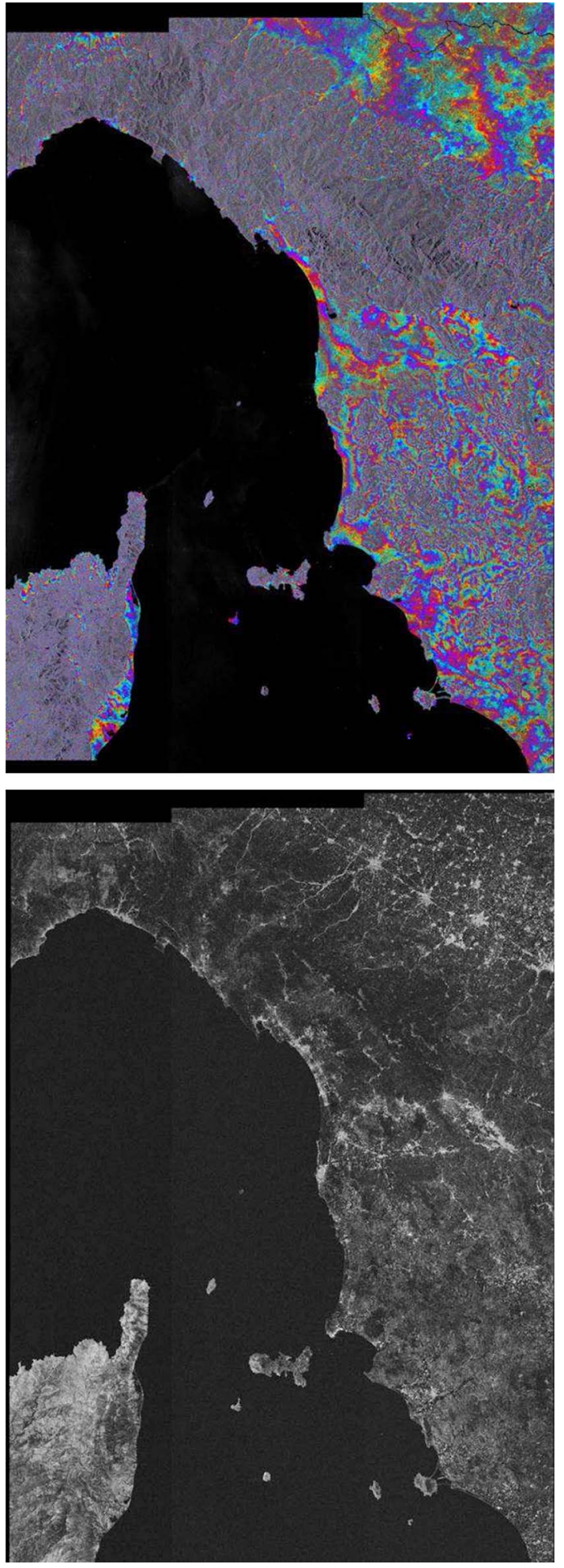

Fig. 11. Mosaic of the first S-1A TOPS interferogram (two slices) in IW mode around the Gulf of Genoa, Italy, acquired on August 7 and 19. Interferometric phase overlaid with the SAR amplitude (top) and coherence (bottom). Range in horizontal direction. Range extension, $250 \mathrm{~km}$; azimuth extension, $340 \mathrm{~km}$.

of the S-1 mission. A total of seven slices has been used for the mosaic, which corresponds to approximately 30 ERS full scenes. The residual fringes that can be observed are probably due to atmospheric disturbances.
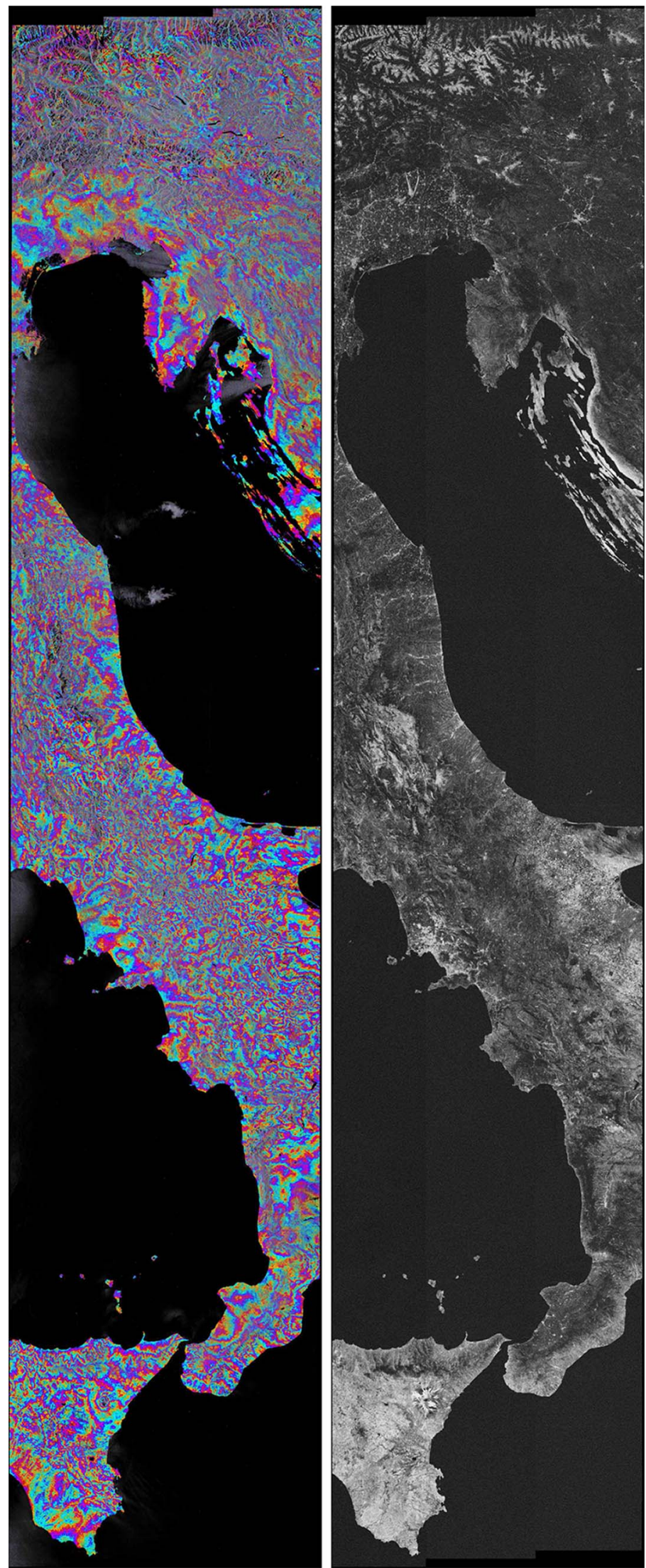

Fig. 12. Mosaic of seven IW slice interferograms in IW mode acquired on August 9 and 21. Interferometric phase, after a simulated phase from a SRTM DEM has been removed, overlaid with the SAR amplitude (left) and coherence (right). Range in horizontal direction. Range extension $250 \mathrm{~km}$; Azimuth extension $1200 \mathrm{~km}$. 

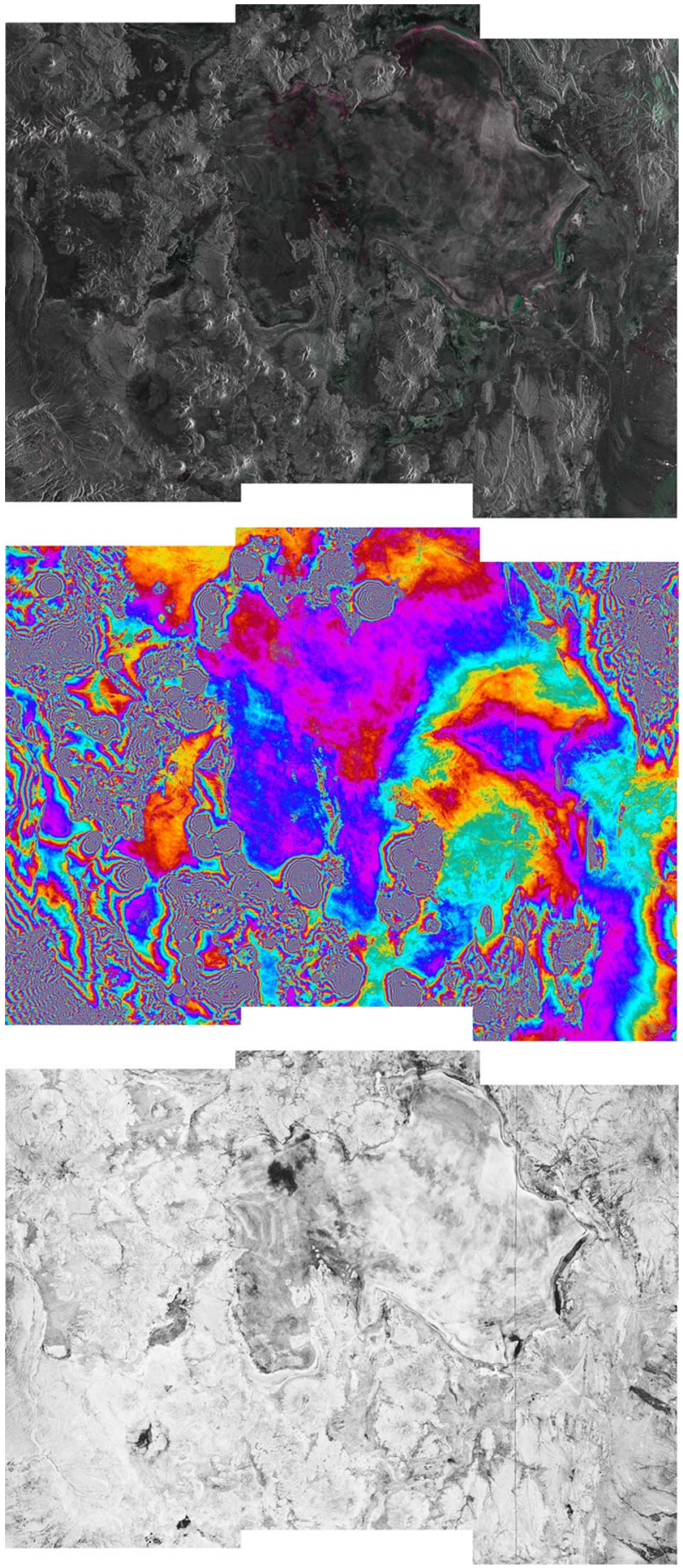

Fig. 13. Interferometric acquisitions in IW mode, descending geometry on December 3 and 27 over Salar de Uyuni, Bolivia. The effective baseline is $108 \mathrm{~m}$, corresponding to a phase cycle of $146 \mathrm{~m}$. Interferometric phase (top), differential phase-SRTM phase subtracted (middle), and coherence (bottom). Range in horizontal direction, increasing to the left. Range extension $250 \mathrm{~km}$, azimuth extension $170 \mathrm{~km}$. The images are in slant-range coordinates but oriented geographically.

\section{INTERFEROMETRIC EVALUATION}

Here, an interferometric evaluation of S-1A data is carried out. A highly coherent interferometric pair in IW mode covering Salar de Uyuni, Bolivia, is first analyzed in terms of Doppler spectrum (deramping), burst synchronization, and coregistration. The spectral shift was already analyzed in Section III-E.

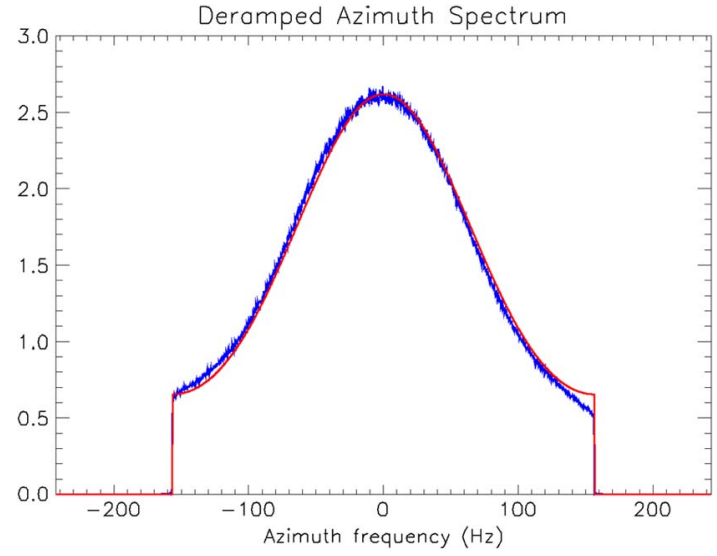

Fig. 14. Deramped Doppler spectrum averaged over range for an S-1A acquisition over Bolivia on December 3, 2014. The blue curve results from a databased estimate, whereas the red curve corresponds to the annotated Hamming weighting window. The fourth burst of the IW2 subswath was selected.

Later, an evaluation of the along-track shifts employing two types of orbits, i.e., precise and restituted, is provided.

The Uyuni salt lake is the world's largest salt flat covering $10582 \mathrm{~km}^{2}\left(4086 \mathrm{mi}^{2}\right)$. It is located in Potosí, near the crest of the Andes, at an elevation of $3656 \mathrm{~m}$ above mean sea level. The Salar is covered by a few meters of salt crust, which has an extraordinary flatness. The datatakes were acquired in descending geometry on December 3 and 27, 2014, with VV polarization. The L1 products were generated with Processor version IPFV241. The effective baseline is about $108 \mathrm{~m}$, corresponding to a phase cycle of approximately $146 \mathrm{~m}$. Fig. 13 shows a multitemporal composite of the amplitudes, the interferometric phase, and the coherence. The observed slow variation of the interferometric phase over the salt flat is possibly due to atmospheric effects.

\section{A. Deramping}

To illustrate the spectral properties of the $S-1$ bursts, a plot of the deramped Doppler spectrum of a burst from IW2 is provided in Fig. 14. The deramping function has been applied to the burst, and an azimuth fast Fourier transform performed followed by an averaging over range. The plot demonstrates that, after deramping, the spectrum is confined within the expected $313 \mathrm{~Hz}$ bandwidth of IW2. The blue curve corresponds to the estimated Doppler spectrum, whereas the red curve indicates the theoretical Hamming weighting window applied by the SAR processor.

\section{B. Burst Synchronization}

Regarding burst synchronization, the along-track position mismatching in the middle of the scene is $0.12 \mathrm{~ms}$, equivalent to $0.82 \mathrm{~m}$ on the ground. The platform Doppler centroids in the middle of the scene were approximately 2 and $4 \mathrm{~Hz}$ for the first and second acquisitions, respectively, indicating excellent burst synchronization characteristics. Fig. 9(a), (c), and (e) shows the variation in range of the Doppler centroid frequencies for all three subswaths. 
TABLE III

Retrieved AZIMUth ShIFTS, IN PiXels AND CENTIMETERs, For EACH Subs WATH OF THE ACQUiSition OvER SALAR DE UYUNI

\begin{tabular}{|c|c|c|c|}
\hline az. retrieved shift & IW1 & IW2 & IW3 \\
\hline az. shift (pix) & -0.00730 & -0.00704 & -0.00713 \\
\hline az. shift (cm) & -10.2677 & -9.9108 & -10.0274 \\
\hline
\end{tabular}

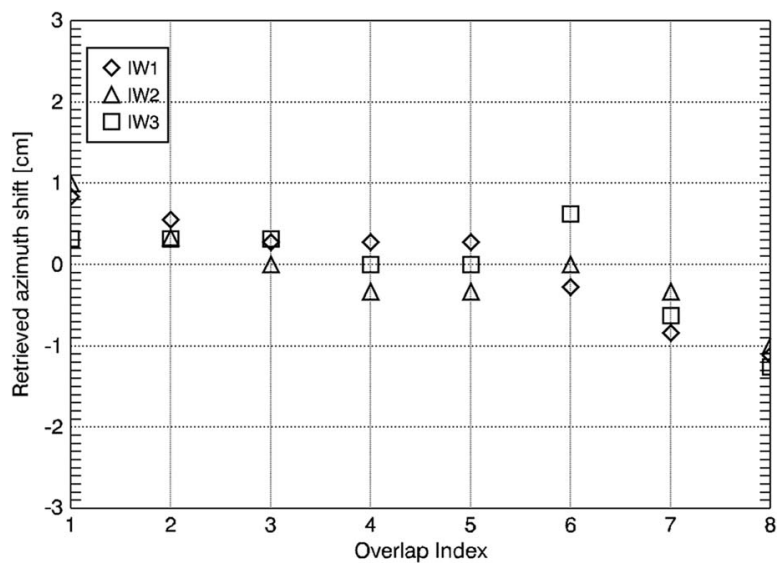

Fig. 15. Residual azimuth shift measured in the overlap areas within each subswath of the slice.

\section{Coregistration Analysis}

The consistency of the retrieved azimuth shift for each overlap area within every subswath is analyzed here. Precise orbits were used for interferometric processing. ESD was used to retrieve the fine residual azimuth shift of approximately $-10 \mathrm{~cm}$. The estimation of the residual azimuth shift was carried out independently for each subswath in order to check the consistency between all three subswaths. Table III summarizes the retrieved shift for each subswath showing differences of a few millimeters, which are negligible. This indicates that a global azimuth shift can be estimated by considering all overlap areas of all three subswaths.

We can also check for the consistency of the residual azimuth shift within each subswath by reestimating the azimuth shift per overlap region after correction of the azimuth shifts given in Table III. Fig. 15 shows this residual azimuth shift for all three subswaths. Observe that the curves are centered around zero. The standard deviation of the shifts for each subswath is $6.8 \mathrm{~mm}$ for IW1, $5.8 \mathrm{~mm}$ for IW2, and $6.2 \mathrm{~mm}$ for IW3.

The reader should keep in mind that an azimuth shift error of $1.2 \mathrm{~cm}$ corresponds to a phase variation along the burst of approx $3.6^{\circ}$. With the plot of Fig. 15, it can be concluded that the remaining phase error is below this value.

\section{Common Doppler Bandwidth}

The common Doppler bandwidth is evaluated here with a stack of acquisitions over Mexico City in descending geometry. The images were acquired between October 3, 2014 and January 1, 2015. The acquisition on December 2, 2014, has been selected as the master. Fig 16 shows the common Doppler bandwidth for each acquisition pair for each subswath. The available Doppler bandwidth is indicated with a horizontal line for each subswath. The common bandwidth is above $95 \%$ of the

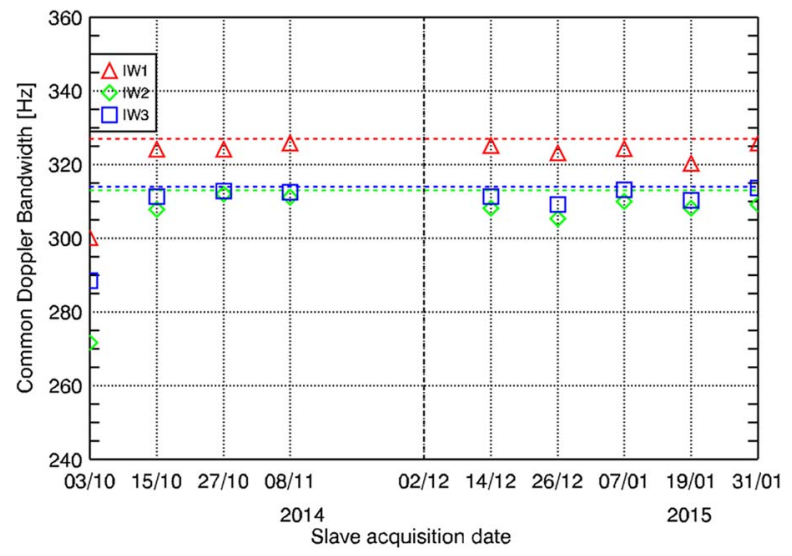

Fig. 16. Assessment of the common Doppler bandwidth. A stack of a total of 10 acquisitions over Mexico City in IW mode has been analyzed. The acquisition on December 2, 2014, has been chosen as master (dashed vertical line). The available Doppler bandwidth is indicated with a horizontal line. The common Doppler bandwidth for each acquisition pair for each subswath is shown.

available bandwidth for all pairs, except for the first acquisition, which was acquired in an early phase, and an issue with the attitude steering was still not solved. This analysis indicates excellent burst synchronization and attitude steering of the system, concluding that the azimuth spectral shift filtering could be skipped without having a significant coherence drop.

\section{E. Along-Track Shifts}

Since very sensitive measurements in the along-track direction can be performed with TOPS acquisitions, we can very accurately evaluate the performance of the precise and restituted orbits in this direction. The analysis can be done in time, by analyzing the differential azimuth orbital error for a stack of images, and in space, by analyzing the consistency of the differential azimuth orbital error for consecutive slices within a datatake. The ESD technique is exploited for this purpose, which measures the differential azimuth shift in the burst overlap areas between the slave image(s) and the master image.

1) Temporal Consistency: The same data set of ten S-1A slices acquired over Mexico is analyzed here. The residual along-track shift has been measured employing ESD technique, which not only accounts for the orbital timing error but also for timing error of the SAR instrument, SAR processing effects, ionospheric effects, and geodynamic effects. We considered the gravitational effects exerted by the Moon and the Sun on the Earth's crust (solid Earth tides), which can reach a few centimeters in the horizontal direction [40].

Fig. 17 shows the retrieved differential orbit error in the along-track direction for the precise and restituted orbits, on the order of a few centimeters. We have to be careful when drawing conclusions from this plot. The retrieved azimuth shift is differential between the slave acquisitions with respect to the master acquisition. This means that a possible bias in the along-track shift cannot be determined since the along-track orbital error of the master acquisition is also a realization of the same process. We are interested in the dispersion of the values and provide the standard deviation, which is $4.42 \mathrm{~cm}$ for the precise orbit and $2.52 \mathrm{~cm}$ for the restituted orbit. From 


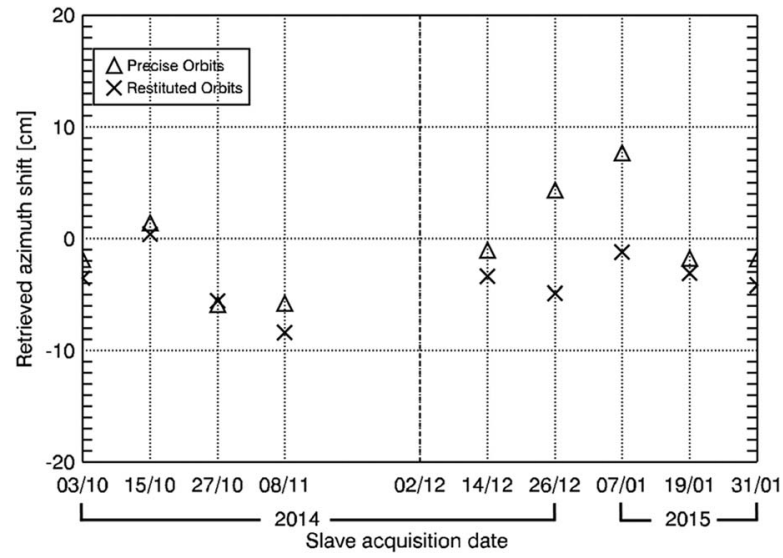

Fig. 17. Assessment of the differential along-track accuracy of the precise and restituted orbits over time. A stack of a total of ten acquisitions over Mexico City in IW mode has been analyzed. The acquisition on December 2, 2014 has been chosen as master (dashed line). The standard deviation of the precise orbit is 4.42 and $2.51 \mathrm{~cm}$ for the restituted orbit. The overlap areas within IW2 have been used for the measurement. The differential solid Earth tides were considered.

the specifications, the nominal (1-D) standard deviation of the azimuth orbital error - assuming an isotropic distribution of the error in the different components (along-track and crosstrack for the restituted orbit; radial, along-track and cross-track for the precise orbit) - would be $2.88 \mathrm{~cm}$ for the precise orbit, and $7.07 \mathrm{~cm}$ for the restituted orbit (assuming that the standard deviation is the same for each direction). We conclude that the measured standard deviation of the precise orbit is very similar to the expected one from the specifications. However, we observe that the measured standard deviation of the restituted orbit is surprisingly better than nominal and paradoxically even better than the one obtained with the precise orbits in this particular data set.

2) Spatial Consistency: We have selected a datatake pair acquired over Europe in descending geometry, composed of six slices. The datatakes were acquired on December 21, 2014 and January 2, 2015 and include the area from Hamburg, Germany to Genoa, Italy. Fig. 18 shows the estimated differential orbit error in the along-track direction for the precise and the restituted orbits, to be around $-4.7 \mathrm{~cm}$ when using precise orbits, and $-4.6 \mathrm{~cm}$ when using restituted orbits. The standard deviation of the differential orbit error in the along-track direction retrieved with the precise orbit is $9.5 \mathrm{~mm}$ and $7.4 \mathrm{~mm}$ for the restituted orbit. The spatial stability of the differential orbit error for both types of orbits is below $1 \mathrm{~cm}$. This information is of valuable interest when working with scenarios that present deformation in the along-track direction. The shift to be corrected can be retrieved from a slice not affected by deformation and applied to the slice(s), experiencing deformation.

\section{CONCLUSION}

In this paper, a guideline on how to process interferometric S-1 data in TOPS mode has been provided. The analysis has been performed for IW mode, i.e., the standard mode over land. A brief description of the S-1 IW TOPS mode, including spectral properties, burst synchronization aspects, and needed coregistration requirements, has been provided. The central

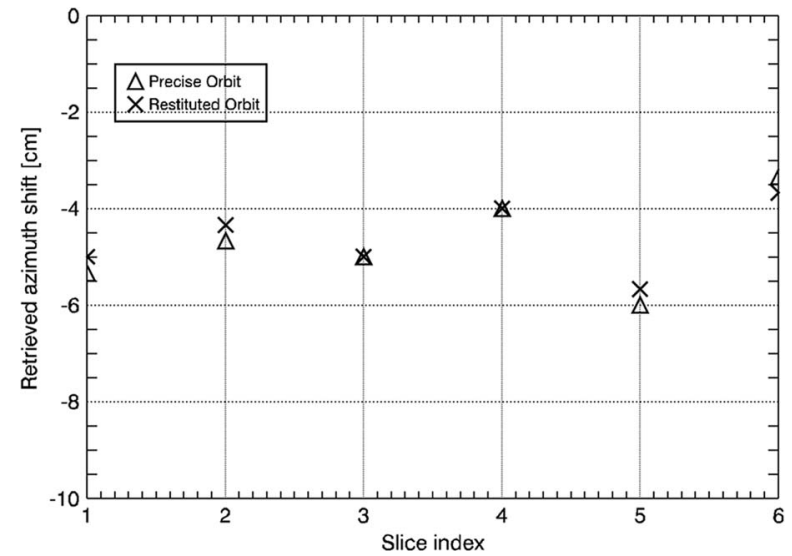

Fig. 18. Assessment of the differential along-track accuracy of the precise and restituted orbits along a datatake. Six slices of a datatake over Germany in IW mode have been employed. The acquisitions were made on December 21, 2014 and January 2, 2015. The standard deviation of the precise orbit is 9.5 and $7.4 \mathrm{~mm}$ for the restituted orbit. The overlap areas within IW2 have been used for the measurement.

part of this paper focused on interferometric processing, where aspects such spectral shift filtering, interpolation, and coregistration were discussed. The adopted strategy for coregistration is based on a geometric prediction of the shifts employing orbit information and an external DEM, followed by a refinement of the azimuth shifts using ESD [9] and a refinement of the range shifts using incoherent cross-correlation. We have proposed a pixelwise estimation of the azimuth shift, considering the shift and the local Doppler centroid frequency differences for each pixel in the overlap area. Other aspects such as data importing, preparation, and mosaicking of interferometric results have been also discussed. First S-1A interferograms in IW TOPS mode acquired during the commissioning phase have been shown, including an interferogram of a datatake over Italy, reaching from Sicily to the Alps that demonstrates the high mapping capabilities of the S-1 mission. An interferometric evaluation with highly coherent data over Salar de Uyuni has been provided, where a quality analysis of the azimuth shifts, both within and between subswaths, indicates high phase stability. We conclude that the azimuth shift can be retrieved globally by employing the overlap areas of all three subswaths. An evaluation of a stack of acquisitions over Mexico City has shown that the common Doppler bandwidth is above 95\% of the available bandwidth, indicating very good burst synchronization and attitude steering of the system. An evaluation of the along-track shifts employing ESD shows that the precise and restituted orbits provide similar accuracy in the alongtrack direction. Moreover, by compensating solid Earth tides, an assessment of the performance of both types of orbits has been provided. The spatial stability of the azimuth shifts for several slices of a datatake has also been provided, showing that the retrieved differential orbital error possesses high spatial stability.

\section{APPENDIX}

L1 B Product Description Format (XML, TIFF, ETC.)

Focused S-1 SAR data products are available in the socalled "SAFE" container format comprising the binary raster 
data as Tagged Image File Format (TIFF) files and various annotation information in eXtensible Markup Language (XML) files. Combinations of transmit/receive polarizations are termed "channels". For the TOPS modes, i.e., IW and EW, both the subswath index and polarization are considered channels. The channels of a product are delivered as separate sets within a SAFE container consisting of a TIFF file with its corresponding XML files. This information effectively provides a mask for the valid/invalid samples (pixels) in the SLC data product. Technically, the TIFF files are actually GeoTIFF files containing specific geo-information tags, and the TIFF file sizes commonly exceed $4 \mathrm{~GB}$, thus requiring the Big[Geo]TIFF format. Handling of classic TIFF and BigTIFF files, as well as automatic conversion between binary formats of different hardware platforms, is accomplished by the open-source libTIFF library (4.0.3). Within these (Big) GeoTIFF files, the binary raster data are stored pixel-interleaved with pixels from near range to far range in a line. Lines are stored from early azimuth to late azimuth. The complex-valued L1 data pixels consist of two 16-bit signed integers representing the real and imaginary parts. For the TOPS modes, the bursts are stored in the TIFF of a channel as a series of patches with the same number of lines per burst that can be obtained from the XML annotation. For every such line in every burst, there is also an indication given in the XML annotation for the offset of the first and of the last valid sample or whether all samples in the line are valid. The XML files contain general information about the acquisition parameters and data-specific information for SAR signal processing in the form of polynomial coefficients. Orbital state vectors of the sensor platform that can be used for quicklook purposes are already included in the SAFE containers. Restituted orbital state vector products are available soon after the acquisition. Precise orbit data are available 20 days after the acquisition. The restituted and precise orbit products can be downloaded as separate products in XML format from the S-1 Quality Control webpage [41].

\section{ACKNOWLEDGMENT}

The authors would like to thank N. Miranda from the European Space Agency for his support.

\section{REFERENCES}

[1] Copernicus Programme. [Online]. Available: http://www.copernicus.eu

[2] R. Torres et al., "GMES Sentinel-1 mission," Remote Sens. Environ., vol. 120, pp. 9-24, May 2012.

[3] F. De Zan and A. Monti Guarnieri, "TOPSAR: Terrain observation by progressive scans," IEEE Trans. Geosci. Remote Sens., vol. 44, no. 9, pp. 2352-2360, Sep. 2006.

[4] D. Geudtner, R. Torres, P. Snoeij, M. Davidson, and B. Rommen, "Sentinel-1 system capabilities and applications," in Proc. IEEE IGARSS, Jul. 2014, pp. 1457-1460.

[5] F. De Zan, A. M. Guarnieri, F. Rocca, and S. Tebaldini, "Sentinel-1 radar interferometry applications," in Proc. 7th EUSAR, Jun. 2008, pp. 1-4.

[6] A. Meta, J. Mittermayer, P. Prats, R. Scheiber, and U. Steinbrecher, "TOPS imaging with TerraSAR-X: Mode design and performance analysis," IEEE Trans. Geosci. Remote Sens., vol. 48, no. 2, pp. 759-769, Feb. 2010.

[7] R. Scheiber et al., "Sentinel-1 imaging performance verification with TerraSAR-X," in Proc. 8th EUSAR, Jun. 2010, pp. 1-4.

[8] J. Mittermayer et al., "TOPS Sentinel-1 and TerraSAR-X processor comparison based on simulated data," in Proc. 8th EUSAR, Jun. 2010, pp. $1-4$.
[9] P. Prats-Iraola, R. Scheiber, L. Marotti, S. Wollstadt, and A. Reigber, "TOPS interferometry with TerraSAR-X," IEEE Trans. Geosci. Remote Sens., vol. 50, no. 8, pp. 3179-3188, Aug. 2012.

[10] R. Scheiber, M. Jäger, P. Prats-Iraola, F. De Zan, and D. Geudtner, "Speckle tracking and interferometric processing of TerraSAR-X TOPS data for mapping nonstationary scenarios," IEEE J. Sel. Topics Appl. Earth Observ. Remote Sens., vol. 8, no. 4, pp. 1709-1720, Apr. 2014.

[11] G. Davidson, V. Mantle, B. Rabus, D. Williams, and D. Geudtner, "Implementation of TOPS mode on RADARSAT-2 in support of the Sentinel-1 mission," presented at the ESA Living Planet Symp., Edinburgh, U.K., Sep. 2013.

[12] D. Geudtner, "Implementation of the TOPS mode on RADARSAT-2 in support of the Copernicus Sentinel-1 mission: RADARSAT-2 TOPS SAR Interferometry (InSAR) scene pair data acquisitions," European Space Agency, Paris, France, Tech. Rep. ESA-S1-TN-ESA-SY-0452, 2014.

[13] P. Prats, R. Scheiber, J. Mittermayer, A. Meta, and A. Moreira, "Processing of sliding spotlight and TOPS SAR data using baseband azimuth scaling," IEEE Trans. Geosci. Remote Sens., vol. 48, no. 2, pp. 770-780, Feb. 2010.

[14] M. Rodriguez-Cassola et al., "Doppler-related distortions in TOPS SAR images," IEEE Trans. Geosci. Remote Sens., vol. 53, no. 1, pp. 25-35, Jan. 2015.

[15] W. Xu, P. Huang, Y. Deng, J. Sun, and X. Shang, "An efficient approach with scaling factors for TOPS-Mode SAR data focusing," IEEE Geosci. Remote Sens. Lett., vol. 8, no. 5, pp. 929-933, Sep. 2011.

[16] G. Engen and Y. Larsen, "Efficient full aperture processing of TOPS mode data using the moving band chirp Z-transform," IEEE Trans. Geosci. Remote Sens., vol. 49, no. 10, pp. 3688-3693, Oct. 2011.

[17] G. Sun et al., "Sliding spotlight and TOPS SAR data processing without subaperture," IEEE Geosci. Remote Sens. Lett., vol. 8, no. 6, pp. 1036-1040, Nov. 2011.

[18] J. Holzner and R. Bamler, "Burst-mode and ScanSAR interferometry," IEEE Trans. Geosci. Remote Sens., vol. 40, no. 9, pp. 1917-1934, Sep. 2002.

[19] F. Rodriguez Gonzalez, N. Adam, A. Parizzi, and R. Brcic, "The Integrated Wide Area Processor (IWAP): A processor for wide area persistent scatterer interferometry," in Proc. ESA Living Planet, Sep. 2013, p. 353.

[20] P. Prats et al., "TAXI: A versatile processing chain for experimental TanDEM-X product evaluation," in Proc. IEEE IGARSS, Honolulu, HI, USA, Jul. 25-30, 2010, pp. 4059-4062.

[21] C. Herbert, C. Thain, D. Ramsbottom, P. Lim, and T. Wong, "Sentinel-1 product definition," Macdonald, Dettwiler Assoc. Ltd., Richmond, BC, Canada, Tech. Rep. MDA S1-RS-MDA-52-7440, Mar. 28, 2014.

[22] P. Prats-Iraola et al., "Role of the orbital tube in interferometric spaceborne SAR missions," IEEE Geosci. Remote Sens. Lett., vol. 12, no. 7, pp. 1486-1490, Jul. 2015.

[23] R. Bamler and D. Just, "Phase statistics of interferograms with application to synthetic aperture radar," Appl. Opt., vol. 33, no. 20, pp. 4361-4368, Jul. 1994.

[24] M. Bara, R. Scheiber, and A. Broquetas, "Interferometric SAR signal analysis in the presence of squint," in Proc. IEEE IGARSS, 1999, vol. 1, pp. 257-259.

[25] R. Scheiber and A. Moreira, "Coregistration of interferometric SAR images using spectral diversity," IEEE Trans. Geosci. Remote Sens., vol. 38, no. 5, pp. 2179-2191, Sep. 2000.

[26] N. Miranda, "Definition of the TOPS SLC deramping function for products generated by the S-1 IPF," Eur. Space Agency, Paris, France, Tech. Rep., 2014.

[27] R. Hanssen and R. Bamler, "Evaluation of interpolation kernels for SAR interferometry," IEEE Trans. Geosci. Remote Sens., vol. 37, no. 1, pp. 318-321, Jan. 1999.

[28] E. Sansosti, P. Berardino, M. Manunta, F. Serafino, and G. Fornaro, "Geometrical SAR image registration," IEEE Trans. Geosci. Remote Sens., vol. 44, no. 10, pp. 2861-2870, Oct. 2006.

[29] Sentinels POD team, "Sentinels POD service file format specifications," European Space Agency, Paris, France, Tech. Rep. GMES-GSEG-EOPGFS-10-0075, 2013.

[30] F. De Zan, P. Prats-Iraola, R. Scheiber, and A. Rucci, "Interferometry with TOPS: Coregistration and azimuth shifts," in Proc. 10th EUSAR, Jun. 2014, pp. 1-4.

[31] R. Bamler and M. Eineder, "Accuracy of differential shift estimation by correlation and split-bandwidth interferometry for wideband and delta-k SAR systems," IEEE Geosci. Remote Sens. Lett., vol. 2, no. 2, pp. 151-155, Apr. 2005.

[32] N. Yague-Martinez, F. Rodriguez-Gonzalez, U. Balss, H. Breit, and T. Fritz, "TerraSAR-X TOPS, ScanSAR and WideScanSAR interferometric processing," in Proc. 10th EUSAR, Jun. 2014, pp. 1-4. 
[33] F. De Zan, "Accuracy of incoherent speckle tracking for circular Gaussian Signals," IEEE Geosci. Remote Sens. Lett., vol. 11, no. 1, pp. 264-267, Jan. 2014.

[34] A. Parizzi, X. Y. Cong, and M. Eineder, "First results from multifrequency interferometry. A comparison of different decorrelation time constants at L, C and X band," in Proc. Fringe Workshop, 2009, pp. 2-6.

[35] N. Sakar, R. Brcic, F. Rodriguez-Gonzalez, and N. Yague-Martinez, "Advanced co-registration method for TOPSAR mode," in Proc. IEEE IGARSS, Jul. 2015, pp. 5240-5243.

[36] S. Mancon, A. Monti Guarnieri, and S. Tebaldini, "Sentinel-1 precise orbit calibration and validation," in Proc. FRINGE, Jun. 2015, pp. 1-4.

[37] F. De Zan, P. Prats-Iraola, and M. Rodriguez-Cassola, "On the dependence of delta-k efficiency on multilooking," IEEE Trans. Geosci. Remote Sens., vol. 12, no. 8, pp. 1745-1749, Aug. 2015.

[38] R. Bamler and P. Hartl, "Synthetic aperture radar interferometry," Inv. Prob., vol. 14, pp. R1-R54, 1998.

[39] M. Eineder, N. Adam, R. Bamler, N. Yague-Martinez, and H. Breit, "Spaceborne spotlight SAR interferometry with TerraSAR-X," IEEE Trans. Geosci. Remote Sens., vol. 47, no. 5, pp. 1524-1535, May 2009.

[40] M. Eineder, C. Minet, P. Steigenberger, Xiaoying Cong, and T. Fritz, "Imaging geodesy-Toward centimeter-level ranging accuracy with TerraSAR-X," IEEE Trans. Geosci. Remote Sens., vol. 49, no. 2, pp. 661-671, Feb. 2011.

[41] Sentinel-1 Quality Control. [Online]. Available: https://qc.sentinel1.eo. esa.int/

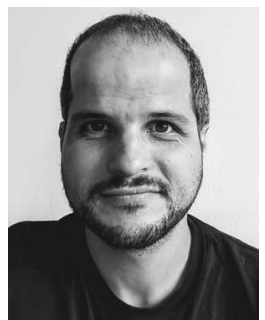

Néstor Yagüe-Martínez received the Ingeniero degree in telecommunications engineering from Universitat Politècnica de València (UPV), Valencia, Spain, in 2004

From 2006 to 2015 he was with the Chair for Remote Sensing Technology, Technische Universität München (TUM), Munich, Germany, and also with the Remote Sensing Technology Institute, German Aerospace Center (DLR), Oberpfaffenhofen, Germany, where he worked in the development of the operational interferometric processor of the TanDEM-X mission. He spent a year from 2011 to 2012 at Starlab Barcelona S.L. working on along-track Interferometry for ocean surface currents retrieval. In 2015 he joined the Multimodal Algorithms Group, Microwaves and Radar Institute, DLR, Oberpfaffenhofen. His research interests include SAR processing, SAR interferometry, and differential SAR interferometry.

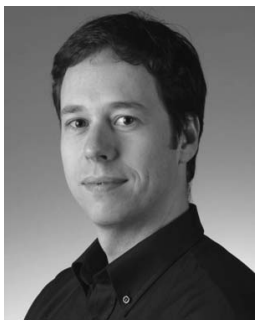

Pau Prats-Iraola (S'03-M'06-SM'13) was born in Madrid, Spain, in 1977. He received the Ingeniero degree and Ph.D. degree in telecommunications engineering from Universitat Politècnica de Catalunya (UPC), Barcelona, Spain, in 2001 and 2006, respectively.

In 2001, he was a Research Assistant with the Institute of Geomatics, Barcelona. In 2002, he was with the Department of Signal Theory and Communications, UPC, where he worked in the field of airborne repeat-pass interferometry and airborne differential SAR interferometry. From December 2002 to August 2006, he was an Assistant Professor with the Department of Telecommunications and Systems Engineering, Universitat Autònoma de Barcelona, Barcelona. In 2006, he joined the Microwaves and Radar Institute, German Aerospace Center (DLR), Wessling, Germany, where, since 2009, he has been the Head of the Multimodal Algorithms Group. His research interests include high-resolution airborne/spaceborne SAR processing and SAR interferometry.

Dr. Prats-Iraola received the First Prize in the Student Paper Competition of the 2005 IEEE International Geoscience and Remote Sensing Symposium held in Seoul, Korea, and the DLR Science Award in 2012.

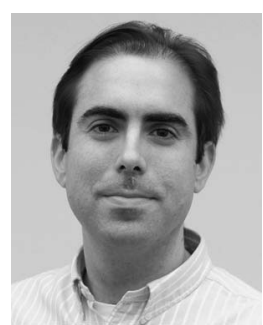

Fernando Rodríguez González received the Ingeniero degree in telecommunications engineering from Universidad Politécnica de Madrid (UPM), Madrid, Spain, and the Diplôme de l'École Polytechnique from École Polytechnique, Palaiseau, France, in 2009.

From 2009 to 2010, he worked at the Chair of Remote Sensing Technology with Technische Universität München, Munich, Germany. In 2010, he joined the Department of SAR Signal Processing, Remote Sensing Technology Institute, German Aerospace Center (DLR), Wessling, Germany. He has worked in the development of the operational interferometric processor of the TanDEM-X mission. His research interests include SAR interferometry, persistent scatterer interferometry, and SAR stereo radargrammetry.

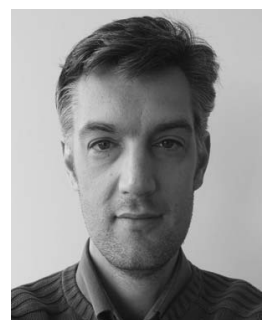

Ramon Brcic (M'03) received the B.Eng. (Hons. 1) degree in aerospace avionics from the Queensland University of Technology, Brisbane, Australia, in 1998 and the Ph.D. degree in electrical engineering from Curtin University of Technology, Perth, Australia, in 2003.

From 2003 to 2007, he was a Research Associate with the Signal Processing Group, Technische Universität Darmstadt, Darmstadt, Germany. Since 2008, he has been with the Department of SAR Signal Processing, Remote Sensing Technology Institute, German Aerospace Center (DLR), Wessling, Germany, as a Research Scientist. His current research interests include interferometric SAR and persistent scatterer interferometry.

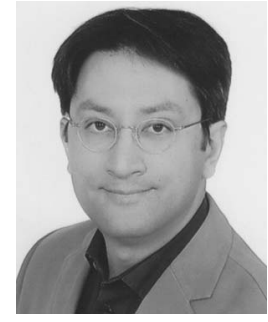

Robert Shau (M'01-SM'13) received the Diploma and the Doctorate degrees in physics in the field of semiconductor laser diodes from Technische Universität München (TUM), Munich, Germany, in 1998 and 2005, respectively.

From 1998 to 2003, he worked at TUM on the development of optical gain material, device structure, and fabrication, and on prototyping, characterization, and application development, of vertical-cavity surface-emitting laser diodes based on indium phosphide in the near infrared. From 2002 to 2010, he worked at a spin-off company cofounded by him on the commercialization of these devices and invented a design for tunable laser applications with low fringe noise. He continued working as a Technology Consultant. Since 2013, he has been with German Aerospace Center, Wessling, Germany and is currently working in the field of persistent scatterer interferometry. 


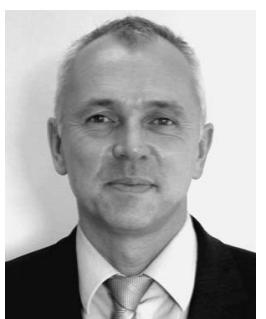

Dirk Geudtner received the M.S. degree in geophysics from Freiberg University of Mining and Technology, Freiberg, Germany, in 1991 and the $\mathrm{Ph} . \mathrm{D}$. degree in InSAR from the University of Stuttgart, Stuttgart, Germany, in 1995.

In 1991, he joined the DLR, where he worked as a SAR Scientist on the development of new methods for satellite InSAR data processing and analysis. In 1994, he joined the German Research Center for Geosciences and became responsible for the development of geoscientific applications of satellite InSAR techniques. From 1995 to 1997, he was a Visiting Scientist with the Canada Centre for Remote Sensing (CCRS), working on differential InSAR for monitoring glacier and ice motion. In 1997, he joined the Microwaves and Radar Institute, DLR, where he developed InSAR calibration concepts for the X-SAR system on the Space Shuttle Radar Topography Mission (SRTM). During SRTM operations, he was the Lead Engineer for the in-orbit X-SAR interferometric antenna beam alignment at the NASA Payload Operations Control Center, Houston, TX, USA. From 2000 and 2002, with a Research Grant from the German Humboldt Foundation. He was a Visiting Scientist at CCRS, working on new methods for the interferometric processing of Canada's RADARSAT- 1 data and for the polarimetric calibration of RADARSAT- 2 data. From 2002 and 2008, during a six-year assignment to the Canadian Space Agency, he worked as key SAR Scientist/Engineer on feasibility studies for future satellite SAR missions focusing particularly on CSA's RADARSAT Constellation Mission. For the RCM project, he was entrusted to lead the external Canadian SAR expert Image Quality and Science Advisory Group. After returning back to DLR in 2008, he significantly contributed to the TerraSAR-X2 feasibility study and the analysis of methods for orbital baseline calibration for the TanDEM-X mission. From 2009 to 2011, he worked as a Scientific Advisor (Seconded National Expert) at the European Commission's Global Monitoring for Environment and Security (GMES) Bureau, where he supported the analysis of the GMES space component and was also responsible for the implementation of the GMES marine and climate change monitoring services. Since March 2011, he has been the Head of the Sentinel-1 Mission and Systems Section at the European Space Agency. In this role, he is responsible for the compliance of the SAR system performance with user/science requirements, the end-to-end SAR system calibration, and the initiation and technical supervision of SAR system and related application studies.

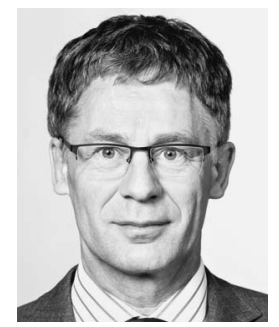

Michael Eineder (SM'01) received the Diploma degree in electrical engineering from Technische Universität München (TUM), Munich, Germany, in 1990 and the Dr. rer. Nat. degree from the University of Innsbruck, Innsbruck, Germany, in 2004.

Since 1990, he has been with German Aerospace Center (DLR), Wessling, Germany, where he has worked on a variety of international missions including SIR-C/X-SAR, SRTM/X-SAR in cooperation with NASA, ERS-1 (ESA), TerraSAR-X, and TanDEM-X (Germany). Since 2013, he has been an Honorary Professor with TUM. He is currently the Head of the Department of SAR Signal Processing, Remote Sensing Technology Institute, DLR. His responsibilities encompass the development of SAR and InSAR processing systems for current and future radar missions. Furthermore, he is a Part-Time Lecturer for remote sensing at TUM. His current research interests include future SAR missions and imaging geodesy, an absolute positioning technique exploiting high-resolution SAR.

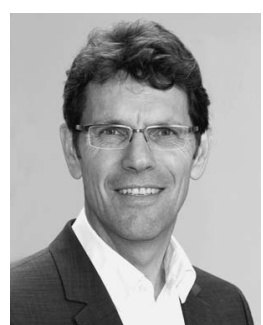

Richard Bamler (M'95-SM'00-F'05) received the Diploma degree in electrical engineering, the Doctorate degree in Engineering, and the Habilitation in signal and systems theory from the Technische Universität München (TUM), Munich, Germany, in 1980, 1986, and 1988, respectively.

From 1981 to 1989 , he worked with TUM on optical signal processing, holography, wave propagation, and tomography. Since 1989, he has been with the German Aerospace Center (DLR), Wessling, Germany, where he is currently the Director of the Remote Sensing Technology Institute. In early 1994, he was a Visiting Scientist with Jet Propulsion Laboratory, in preparation of the SIC-C/X-SAR missions. In 1996, he was a Guest Professor with the University of Innsbruck, Innsbruck, Germany. Since 2003, he has been a Full Professor of remote sensing technology with TUM as a double appointment with his DLR position. His teaching activities include university lectures and courses on signal processing, estimation theory, and SAR. He, his team, and his institute have been working on SAR and optical remote sensing, image analysis and understanding, stereo reconstruction, computer vision, ocean color, passive and active atmospheric sounding, and laboratory spectrometry. They were and are responsible for the development of the operational processors for SIR-C/X-SAR, SRTM, TerraSAR-X, TanDEM-X, Tandem-L, ERS-2/GOME, ENVISAT/SCIAMACHY, MetOp/ GOME-2, Sentinel 5p, EnMAP, etc. He has devised several high-precision algorithms for monostatic and bistatic SAR processing, SAR calibration and product validation, ground moving target indication for traffic monitoring, SAR interferometry, phase unwrapping, persistent scatterer interferometry, differential SAR tomography, and data fusion. His research interests include algorithms for optimum information extraction from remote sensing data with emphasis on SAR, which involves new estimation algorithms, such as sparse reconstruction and compressive sensing. 